\title{
An analysis of the convergence of the direct simulation Monte Carlo method
}

\author{
Cyril Galitzine ${ }^{\mathrm{a}, *}$, Iain D. Boyd ${ }^{\mathrm{a}}$ \\ ${ }^{a}$ University of Michigan, Department of Aerospace Engineering, Ann Arbor, MI 48109, United States
}

\begin{abstract}
In this article, a rigorous framework for the analysis of the convergence of the direct simulation Monte Carlo (DSMC) method is presented. It is applied to the simulation of two test cases: an axisymmetric jet at a Knudsen number of 0.01 and Mach number of 1 and a two-dimensional cylinder flow at a Knudsen of 0.05 and Mach 10. The rate of convergence of sampled quantities is found to be well predicted by an extended form of the Central Limit Theorem that takes into account the correlation of samples but requires the calculation of correlation spectra. A simplified analytical model that does not require correlation spectra is then constructed to model the effect of sample correlation. It is then used to obtain an a priori estimate of the convergence error.
\end{abstract}

Keywords: DSMC, direct simulation Monte Carlo, particle method, error, convergence, correlation

\section{Introduction}

Error in Monte Carlo particle methods, such as e.g. DSMC (direct simulation Monte Carlo), can be defined, in the broadest possible sense, as the difference between the statistical properties of the computational particles and the corresponding moments of the exact solution of the equation being discretized. This error originates from multiple sources which either mitigate or reinforce one another. The first type of error is that introduced by the use of a finite number of samples $k$ to estimate the statistical properties of the particles in the simulation. It will be referred to as the convergence error in this article. Error can also come from the use of finite numerical parameters in the application of the particle method which introduces error due to the numerical scaling (as each computational particle represents $W_{p}$ physical particles, and both time and space are discretized). This error is most commonly due to the use of a too small number of particles $N_{p}$, a too large time step $\Delta t$, or too large cells. This type of error will be termed numerical error in the following. Error can also be due to the inability of the method, in the absence of numerical error, to reproduce the moments of the PDE being discretized. This issue will not be addressed in the present work noting that DSMC, the method used in this article, has been shown to consistently model the homogeneous Boltzmann equation [1] in the limit of $N_{p} \rightarrow \infty$.

Convergence error, the focus of this article, is referred to as the statistical error in [2] and was found in [3] to vary as $A(N \times k)^{-1 / 2}$. The same result was later obtained in [4]. The error was related in [2] to the physical fluctuations in the gas and closed form expressions were obtained in terms of non-dimensional flow numbers for $A$. From an error perspective, the role of sampling in a DSMC simulation is to reduce the convergence error affecting the quantities being sampled for. Despite the previous investigations of the convergence error cited above, there are no clear requirements within the DSMC community as to the number of sampling steps required to obtain accurate or converged results for those sampled properties. Often an arbitrarily large number of samples, e.g. $10^{5}[5]$ is used without further considerations or the sampling period is extended [6] "until the statistical error is small enough". This situation is, in no small part, due

\footnotetext{
* Corresponding author.

Email address: cyrilg@umich.edu (Cyril Galitzine)
} 
to the correlation between samples which prevents the use of the Central Limit Theorem [7]. For instance, the predictions for the statistical error due to finite sampling presented in [2] only holds in the absence of correlation between samples which leads its authors to sample only every 250 time steps in the DSMC simulations conducted to validate them. Many authors also try to reduce the correlation between successive samples by only sampling every few time steps such as [8] or [9] to obtain more independent samples. This shows that the time correlation between samples in DSMC simulations is a well known issue although it has not been accounted for in any existing work about the convergence error. The present article thus aims to derive expressions for the convergence error that take the correlation of samples into account.

The objective of this article is furthermore to present an investigation of the convergence error within the framework of a test case that is sufficiently complex so as to highlight features or phenomena that users of the method are likely to encounter in practice. To accomplish this, instead of using a relatively simple canonical test case such as channel flow [10] [2] [11], two more complex cases are investigated. The first consist of an axisymmetric supersonic jet, which is a configuration of great practical interest to the rarefied gas dynamics community [12]. The second test case is a two-dimensional hypersonic cylinder flow which is often used for numerical studies [13] [14].

In this paper, a rigorous formal definition of the convergence error in Monte Carlo particle simulations is first presented. The goal of the article is to provide an expression for the value of the convergence error that can be used for its a priori determination during the course of the simulation. As previously mentioned, the convergence error is greatly affected by the time correlation between samples. This makes it necessary to quantify the correlation between samples, an issue which is discussed in Section 3. A Central Limit Theorem that takes correlation into account is then introduced. It provides an expression for the aforedefined convergence error in terms of the autocorrelation function of samples. This expression can readily be used "on the fly" during the simulation to assess the convergence of cellwise sampled quantities. Convergence error predictions are then assessed within the framework of the two previously mentioned test cases.

\section{Framework for error analysis}

\subsection{Implementation of the DSMC procedure}

A simple argon gas in a two-dimensional (or axisymmetric) domain $\Omega=\{(x, y)\} \subset \mathbb{R}^{2}$ with velocities in $\mathbb{R}^{3}$ is considered. $\bar{\Omega}$ is decomposed into $N_{c}$ quadrangular cells $\left\{\Omega_{i}\right\}_{i=1}^{N_{c}}$ of respective volume $\left\{V_{i}\right\}_{i=1}^{N_{c}}$ via quadrangulation $\Omega_{h}$ :

$$
\bar{\Omega}=\bigcup_{\Omega_{i} \in \Omega_{h}} \Omega_{i}
$$

such that $\mathrm{V}_{i} \leq h, \forall 1 \leq i \leq N_{c}$. The number density $n_{i}$ in cell $i$ that contains $N_{i}$ particles is obtained with

$$
n_{i}=\frac{W_{p} N_{i}}{V_{i}}
$$

each computational particle representing $W_{p}$ physical particles so that the entire domain contains $N_{p}$ particles. A constant time step $\Delta t$ and scaling factor $W_{p}$ are used throughout the domain. The axisymmetric move procedure detailed in [15] p. 371 is employed for the first test case while a standard Euler explicit scheme is used for the second which is two-dimensional. The standard NTC (No Time Counter) collision scheme [15] is used to calculate the number of potential collisions in each cell:

$$
N_{\mathrm{coll}, i}=\frac{1}{2 \mathrm{~V}_{i}} W_{p} \Delta t N_{i} \bar{N}_{i}(\sigma g)_{\max , i}
$$

where $\bar{N}_{i}$, the average number of particles in the cell, is obtained by an exponential moving average with a relaxation factor of 0.001. Binary elastic collisions are modeled via the variable hard sphere model [15] with a reference diameter of $4.17 \times 10^{-10} \mathrm{~m}$ at $273 \mathrm{~K}$ and a temperature exponent of 0.81 . Further details concerning our implementation of the DSMC procedure can be found in [16]. 


\subsection{Cell and particle based variables}

Any variable characterizing particle $j$ at time $t^{k}$ is denoted by $\mathbf{y}_{j}^{k}$ with $1 \leq j \leq N_{p}$ which can for instance represent its instantaneous position $\overrightarrow{\mathbf{x}}_{j}^{k}$ or velocity $\overrightarrow{\mathbf{v}}_{j}^{k}$. Cell-based quantities are denoted by $y_{i}^{k}$ with $1 \leq i \leq N_{c}$ which designates any variable specific to cell $i$ at time $t^{k}$ such as the number of particles contained in the cell $N_{i}^{k}$. When all particles or all cells of the simulations are simultaneously considered, particle and cell-based quantities can both be represented by a series of vectors, namely $\overrightarrow{\mathbf{y}}^{k}=\left(\mathbf{y}_{1}^{k}, \ldots, \mathbf{y}_{N_{p}}^{k}\right)$ and $\vec{y}^{k}=\left(y_{1}^{k}, \ldots, y_{N_{c}}^{k}\right)$. The average value of $\mathbf{y}$ in cell $\Omega_{i}$ at time step $k$ is naturally defined as:

$$
y_{i}^{k}=\langle\mathbf{y}\rangle_{\Omega_{i}}^{k}=\frac{\sum_{j=1}^{N_{i}^{k}} \mathbf{y}_{j}^{k}}{N_{i}^{k}} .
$$

The transformation of particle-based quantities to cell-based ones can more formally be seen as a linear transformation. To formalize the transformation, an indicator function $\mathbf{1}_{\Omega_{i}}: \mathbf{R}^{2} \rightarrow\{0,1\}$ is defined as

$$
\mathbf{1}_{\Omega_{i}}(\mathbf{p}):= \begin{cases}1 & \text { if } \mathbf{p} \in \Omega_{i} \\ 0 & \text { if } \mathbf{p} \notin \Omega_{i}\end{cases}
$$

which determines whether or not a given particle located at position $\mathbf{p}$ belongs to $\Omega_{i}$. This function can be used to give a more rigorous definition of number density than that of Eq. (2):

$$
n_{i}^{k}=\frac{W_{p} \sum_{j=1}^{N_{p}} \mathbf{1}_{\Omega_{i}}\left(\overrightarrow{\mathbf{x}}_{j}^{k}\right)}{\mathrm{V}_{i}} .
$$

The indicator function can also be used to rewrite Eq. (4) more rigorously as:

$$
y_{i}^{k}=\langle\mathbf{y}\rangle_{\Omega_{i}}^{k}=\frac{\sum_{j=1}^{N_{p}} \mathbf{y}_{j}^{k} \mathbf{1}_{\Omega_{i}}\left(\overrightarrow{\mathbf{x}}_{j}^{k}\right)}{\sum_{j=1}^{N_{p}} \mathbf{1}_{\Omega_{i}}\left(\overrightarrow{\mathbf{x}}_{j}^{k}\right)} .
$$

which shows that the projection of a particle-based variable on the mesh depends on both the position of all particles $\left\{\overrightarrow{\mathbf{x}}_{j}\right\}_{j=1}^{N_{p}}$ and the value of the particular variable $\{\mathbf{y}\}_{j=1}^{N_{p}}$.

\subsection{Statistical estimators}

The stochastic particle system engendered by the DSMC procedure is related in practice to the Boltzmann equation by averaging quantities over multiple realizations (i.e. time steps). This is due to the statistical fluctuations inherent to the DSMC formulation which make individual realizations usually too noisy for practical use. The statistical fluctuations of quantities of the DSMC particle system are themselves tied to those of the actual physical system. For instance, the statistical properties of DSMC particles in a given computational cell are identical to those that are present in the same physical cell. One can, in a sense, view DSMC or other particle methods as numerical experiments where measurements are made and collected to estimate statistical properties. Due to the finite number of samples, the estimation of the true statistical properties of the system is imperfect and leads to what is later termed the convergence error. It is thus appropriate to distinguish between the true statistical properties of the DSMC gas and their estimation obtained through sampling. Let $Y_{i}$ be any cell-based variable, e.g. $N_{i}$, and let $y_{i}^{k}$ designate an observed output of that random variable at time step $k . \theta\left(Y_{i}\right)$ refers to any statistical property of random variable $Y_{i}$ such as for example its first order moment $\mu_{1}$. The estimator of $\theta\left(Y_{i}\right)$ at time step $k$ is denoted by $\hat{\theta}^{k}\left(y_{i}\right)$. Because all the statistical estimators used are consistent, i.e. $\lim _{k \rightarrow \infty} \hat{\theta}^{k}\left(y_{i}\right)=\theta\left(Y_{i}\right)$, in the following, the limit of $\hat{\theta}^{k}\left(y_{i}\right)$ when $k \rightarrow \infty$ will be abbreviated as $\theta\left(Y_{i}\right) . \hat{\theta}^{k}\left(y_{i}\right)$ typically depends on all previously observed samples $\left(y_{i}^{1}, \ldots, y_{i}^{k}\right)$ if sampling occurs at every time step, as is the case in the present work. For instance, the estimator for $\mu_{1}\left(y_{i}\right)=\mathbb{E}\left[Y_{i}\right]$ (often referred to as the sampled value of $y$ ) at time step $k$ is defined by:

$$
\hat{\mu}_{1, i}^{k}(y)=\frac{\sum_{k^{\prime}=1}^{k} y_{i}^{k^{\prime}}}{k},
$$


whereas the following estimator is used for $\sigma^{2}\left(Y_{i}\right)=\operatorname{Var}\left(Y_{i}\right)$ :

$$
\hat{\sigma}_{i}^{2, k}(y)=\hat{\mu}_{1, i}^{k}\left(y^{2}\right)-\left(\hat{\mu}_{1, i}^{k}(y)\right)^{2}=\frac{1}{k} \sum_{k^{\prime}=1}^{k}\left(y_{i}^{k^{\prime}}\right)^{2}-\left(\hat{\mu}_{1, i}^{k}(y)\right)^{2} .
$$

In the case of particle-based variables such as velocities, a different statistical estimator, denoted by $\check{\mu}_{1, i}^{k}(\mathbf{y})$, is used:

$$
\check{\mu}_{1, i}^{k}(\mathbf{y})=\frac{\sum_{k^{\prime}=1}^{k} \sum_{j=1}^{N_{i}^{k^{\prime}}} \mathbf{y}_{j}^{k^{\prime}}}{\sum_{k^{\prime}=1}^{k} N_{i}^{k^{\prime}}} .
$$

The use of this estimator amounts to considering each particle at each time step as a sampled value of the velocity distribution (in the case of velocity). This is indeed consistent with the DSMC formulation that considers particles as indistinguishable and the steady state assumption which considers time steps indistinguishable. Because of the time correlation between time steps, which will be studied in section 3, the samples are, however, not independent which, as will later be seen, reduces the rate of convergence of statistical estimators. The variance of $\mathbf{y}$ is obtained similarly to that of $y$ with $\check{\sigma}_{i}^{2} k(\mathbf{y})=\check{\mu}_{1, i}^{k}\left(\mathbf{y}^{2}\right)-\check{\mu}_{1, i}^{k}(\mathbf{y})^{2}$. To simplify notations in the following, $\check{\mu}$ will be denoted by $\hat{\mu}$ with the understanding that $\check{\mu}$ defined by Eq. (10) is used for all particle-based variables instead of the estimator defined by Eq. (8).

The variance of number density can be calculated from Eq. (2):

$$
\operatorname{Var}\left(n_{i}\right)=\left(\frac{W_{p}}{\mathrm{~V}_{i}}\right)^{2} \operatorname{Var}\left(N_{i}\right)
$$

Because $N_{i}$ follows a Poisson distribution for cells with $N_{i} \gg 1[17]$, $\operatorname{Var}\left(N_{i}\right)=\mathbb{E}\left[N_{i}\right]$, so that

$$
\operatorname{Var}\left(n_{i}\right)=\left(\frac{W_{p}}{\mathrm{~V}_{i}}\right) \mathbb{E}\left[n_{i}\right]
$$

Thus, for a given cell, when $W_{p}$ is varied, $\operatorname{Var}\left(n_{i}\right) \sim W_{p}$. This means that the variance of number density is a quantity that directly depends on the number of particles used in the simulation. Its variance will decrease when the value of $W_{p}$ is reduced (or equivalently when the number of particles is increased). In contrast to that, the variance of a particle-based variable does not depend on the numerical parameters of the simulation. Taking one velocity component as an example, e.g. $\mathbf{y}=V_{x}$, its variance obtained with Eq. (9) is equal to the translational temperature of the particles, both computational and physical, in the cell.

\subsection{Error types}

Multiple sources of error are present in a DSMC simulation. They can be broadly placed into two categories. The first comes from the inadequacy of the DSMC procedure to properly model the Boltzmann equation in the absence of any scaling, that is, in the limit of $N \rightarrow \infty, \Delta t \rightarrow 0, k \rightarrow \infty$ and $h \rightarrow 0$. This can, for instance, be caused by an inadequate pair selection scheme or erroneous boundary conditions. This type of error will not be further considered, noting that DSMC has been proven [1] in the homogeneous case and in the limit of $N \rightarrow \infty$ to "converge" to the Boltzmann equation. This article is concerned with the second type of error inherent to DSMC simulations, those introduced by the use of finite values for $k$. As previously mentioned, the effect of the cell size, $h$, on the error will not be considered in the present work. The total error for statistical estimator $\theta\left(Y_{i}\right)$ of variable $Y_{i}$ at step $t^{k}$ of a simulation conducted with $W_{p}$ and $\Delta t$ is defined as being the difference between statistical estimator $\hat{\theta}^{k}\left(W_{p}, \Delta t\right)$ and $\theta$ obtained in the limit of $W_{p} \rightarrow 1$ and $\Delta t \rightarrow 0$. This total error is itself the result of two distinct inexactitudes which either reinforce or mitigate one another. The first is due to the inability to estimate the true value of an estimator with a finite number of samples and is called the convergence error. It is so named because it decreases, as long as the properties being sampled are stationary, with increasing $k$ as the estimators converge to the actual values of the parameter being estimated. The second, called numerical error, is caused by the use of finite $W_{p}$ and $\Delta t$ values. Precise mathematical definitions are now given for the two aforementioned errors. 


\subsubsection{Convergence Error}

The most natural definition of convergence error $\tilde{\varepsilon}_{i}^{k}$ for estimator $\hat{\theta}^{k}$ for cell $i$ at time step $k$ is:

$$
\tilde{\varepsilon}_{i}^{k}[\theta]=\left|\hat{\theta}_{i}^{k}\left(W_{p}, \Delta t\right)-\lim _{k \rightarrow \infty} \hat{\theta}_{i}^{k}\left(W_{p}, \Delta t\right)\right| .
$$

Because of the stochastic nature of DSMC, the convergence of statistical property estimators is jagged and non-monotonic with the final value being both overshot and undershot multiple times during convergence. Thus, if only a single simulation is considered, the convergence error, as previously, defined does not monotonically decrease with time which makes its study difficult. This in turn suggests that a more probabilistic definition of the convergence error is needed. A natural choice is to define the convergence error $\tilde{\varepsilon}_{i}^{k}$ of estimator $\hat{\theta}_{i}^{k}$ at time step $k$ as the standard deviation of $\hat{\theta}_{i}^{k}$ between multiple simulations conducted with the same set of numerical parameters but different sequences of random numbers. Assuming that $N_{\text {sim }}$ simulations are conducted, each will produce a different value for $\hat{\theta}_{i}^{k}$ which is denoted by $\hat{\theta}_{i}^{k, l}$ with $1 \leq l \leq N_{\text {sim }}$. An ensemble average operator $\langle\cdot\rangle$ between simulations is defined as:

$$
\langle z\rangle^{k} \triangleq \frac{1}{N_{\mathrm{sim}}} \sum_{l=1}^{N_{\mathrm{sim}}} z^{k, l}
$$

for every time step and simulation dependent quantity $z^{k, l}$. Applying the ensemble average operator to estimators $\hat{\theta}_{i}^{k, l}$, its ensemble average at time step k is obtained:

$$
\left\langle\hat{\theta}_{i}\right\rangle^{k}=\frac{1}{N_{\mathrm{sim}}} \sum_{l=1}^{N_{\mathrm{sim}}} \hat{\theta}_{i}^{k, l} .
$$

This in turn is used to calculate the variance of $\left\langle\hat{\theta}_{i}\right\rangle^{k}, \operatorname{Var}\left(\left\langle\hat{\theta}_{i}\right\rangle^{k}\right)$ as:

$$
\operatorname{Var}\left[\left\langle\hat{\theta}_{i}\right\rangle^{k}\right]=\left\langle\hat{\theta}_{i}^{2}\right\rangle^{k}-\left(\left\langle\hat{\theta}_{i}\right\rangle^{k}\right)^{2}
$$

which allows the following definition of the convergence error:

$$
\tilde{\varepsilon}_{i}^{k}[\hat{\theta}]=\sqrt{\operatorname{Var}\left[\left\langle\hat{\theta}_{i}\right\rangle^{k}\right]} .
$$

\subsection{Numerical Error}

Numerical error $\bar{\varepsilon}_{i}$ for estimator $\theta_{i}$ is defined for a given simulation characterized by $\Delta t$ and $W_{p}$ as the difference between $\theta_{i}\left(W_{p}, \Delta t\right)$ and that obtained in the absence of any scaling:

$$
\bar{\varepsilon}_{i}[\theta]=\theta_{i}\left(W_{p}, \Delta t\right)-\lim _{W_{p} \rightarrow 1, \Delta t \rightarrow 0} \theta_{i}\left(W_{p}, \Delta t\right) .
$$

The value of the estimator in the limit is of course inaccessible in practice and must therefore be approximated by that obtained with a reference simulation with finite parameters $W_{p, 0} \ll W_{p}$ and $\Delta t_{0} \ll \Delta t$. The reference parameters have to be chosen so that the numerical error calculated for the test case at hand with the reference simulation is much larger than the true numerical error of the reference simulation, i.e.

$$
\left|\theta_{i}\left(W_{p, 0}, \Delta t_{0}\right)-\lim _{W_{p} \rightarrow 1, \Delta t \rightarrow 0} \theta_{i}\left(W_{p}, \Delta t\right)\right| \ll\left|\theta_{i}\left(W_{p}, \Delta t\right)-\theta_{i}\left(W_{p, 0}, \Delta t_{0}\right)\right| .
$$

This allows Eq. (18) to be approximated as:

$$
\bar{\varepsilon}_{i}[\theta] \approx \theta_{i}\left(W_{p}, \Delta t\right)-\theta_{i}\left(W_{p, 0}, \Delta t_{0}\right) .
$$

We, however, note in passing that the precise definition of this error depends on the collision scheme used. The accuracy of the NTC scheme can, for instance, deteriorate when a too small time step is employed, as it cannot capture the exponential distribution of the mean collision time [18]. The numerical error will not be studied in this article, as its a priori determination for complex simulations, such as those considered in the following, is difficult. 


\section{Analysis and consequences of the correlation between samples}

\subsection{The Central Limit Theorem}

Following the definition of Eq. (17), the convergence error for the sampled mean $\hat{\mu}_{1}^{k}(y)$ is due to the variance of $\hat{\mu}_{1}^{k}(y)$ over multiple simulations. In the case where $\left\{y^{k^{\prime}}\right\}_{k^{\prime}=1}^{k}$ are independent and identically distributed (iid), the Central Limit Theorem (CLT) [7] states that

$$
\sqrt{k}\left(\hat{\mu}_{1}^{k}(y)-\mu(Y)\right) \stackrel{\mathcal{L}}{\rightarrow} \mathcal{N}\left(0, \sigma^{2}(Y)\right)
$$

which implies that for $k \gg 1$

$$
\hat{\mu}_{1}^{k}(y) \sim \mathcal{N}\left(\mu_{1}(Y), \frac{\sigma^{2}(Y)}{k}\right)
$$

so that by the definition of the convergence error, following Eq. (17), for $k \gg 1$ :

$$
\tilde{\varepsilon}^{k}\left[\mu_{1}(y)\right]=\frac{\sigma(Y)}{k^{1 / 2}} .
$$

Eq. (23) gives an expression for the evolution of the convergence error that is valid if $Y$ is an iid variable. This is, however, not generally true for most quantities in a DSMC simulation. $N_{i}^{k+1}$ is for instance not independent of $N_{i}^{k}$ but rather correlated with it, as the movement of particles in and out of cell $i$ is quite limited between time steps. This correlation furthermore extends to previous time steps i.e. $N_{i}^{k-1}, N_{i}^{k-2}, \ldots$ etc. Instantaneous quantities in each cell of the simulation $Y_{i}^{k}$ can thus be viewed as discrete correlated time series.

\subsection{Correlation measure}

\subsubsection{Time correlation of cell-based quantities}

The Central Limit Theorem outlined above is derived under the assumption that random variable $Y$ is independent and identically distributed. It thus cannot be applied in its strongest form to a time-correlated time series. This in turn means that the time correlation of sampled quantities needs to be quantified. The autocovariance function $\gamma_{Y}$ of $Y$ at time $k_{0}$ is defined as:

$$
\gamma_{Y}\left(k_{0}+k, k_{0}\right) \triangleq \operatorname{Cov}\left(Y^{k_{0}}, Y^{k_{0}+k}\right)
$$

with:

$$
\operatorname{Cov}\left(Y^{k_{0}}, Y^{k_{0}+k}\right)=\mathbb{E}\left[\left(Y^{k_{0}+k}-\mathbb{E}\left[Y^{k_{0}+k}\right]\right)\left(Y^{k_{0}}-\mathbb{E}\left[Y^{k_{0}}\right]\right)\right]
$$

For stationary processes (such as instantaneous simulation quantities in the steady state regime), the autocovariance function is independent of time $k_{0}$ and only a function of the time difference $k$, so that we define:

$$
\gamma_{Y}(k)=\gamma_{Y}(k, 0)
$$

noting that from Eq. (25):

$$
\gamma_{Y}(-k)=\gamma_{Y}(k)
$$

The autocorrelation function $(\mathrm{ACF})$ of $Y$ is defined as:

$$
\rho_{Y}(k)=\frac{\gamma_{Y}(k)}{\gamma_{Y}(0)}=\frac{\mathbb{E}\left[Y^{k_{0}+k} Y^{k_{0}+k}\right]-\mathbb{E}^{2}[Y]}{\operatorname{Var}[Y]} .
$$

noting that $\operatorname{Var}[Y]=\gamma_{Y}(0)$. The calculation of the ACF is quite straightforward in practice and only requires the storage of $y$ for the previous $k$ steps. 


\subsection{A modified version of the Central Limit Theorem}

The estimator for $\mu_{1}(y)$ at time step $k, \hat{\mu}_{1}^{k}(y)$ is defined as:

$$
\hat{\mu}_{1}^{k}(y)=\frac{1}{k} \sum_{k^{\prime}=1}^{k} y^{k^{\prime}}
$$

A Central Limit Theorem for $\hat{\mu}_{1}^{k}(y)$ that takes into account the time correlation of $y^{k}$ can be derived [19] [20] by taking the variance of Eq. (29):

$$
\operatorname{Var}\left[\hat{\mu}_{1}^{k}(y)\right]=\frac{1}{k^{2}} \sum_{k^{\prime}=1}^{k} \sum_{k^{\prime \prime}=1}^{k} \operatorname{Cov}\left(y^{k^{\prime}}, y^{k^{\prime \prime}}\right) .
$$

From Eq. (26), $\gamma_{Y}\left(k^{\prime \prime}-k^{\prime}\right)=\operatorname{Cov}\left(y^{k^{\prime}}, y^{k^{\prime \prime}}\right)$ and using the symmetry of the $(k \times k)$ covariance matrix to simplify the double sum:

$$
\operatorname{Var}\left[\hat{\mu}_{1}^{k}(y)\right]=\frac{1}{k^{2}}\left(k \gamma_{Y}(0)+2 \sum_{k^{\prime \prime \prime}=1}^{k-1}\left(k-k^{\prime \prime \prime}\right) \gamma_{Y}\left(k^{\prime \prime \prime}\right)\right)
$$

Assuming that $k \gg k^{\prime \prime \prime}$ and truncating the sum at order $K$ so as to only keep terms with a non-negligible contribution to the series, Eq. (31) can be rewritten as follows

$$
\operatorname{Var}\left[\hat{\mu}_{1}^{k}(y)\right] \approx \frac{\gamma_{Y}(0)}{k}+\frac{2}{k} \sum_{k^{\prime \prime \prime}=1}^{K} \gamma_{Y}\left(k^{\prime \prime \prime}\right)=\frac{\sigma^{2}(y)}{k}+\frac{2}{k} \sum_{k^{\prime \prime \prime}=1}^{K} \gamma_{Y}\left(k^{\prime \prime \prime}\right) \triangleq \frac{\tilde{\sigma}^{2}(y)}{k}
$$

The minimum acceptable value for $\mathrm{K}$ depends on how fast $\gamma_{Y}(k)$ decays to 0 . The variance $\operatorname{Var}\left[\hat{\mu}_{1}^{k}(y)\right]$ will be referred to in the following by $\frac{\tilde{\sigma}^{2}(y)}{k}$. Eq. (32) can thus be seen to give an expression for the convergence error for the sampled mean (defined by Eq. 17), that is

$$
\frac{\tilde{\sigma}(y)}{k^{1 / 2}}=\tilde{\varepsilon}^{k}\left[\mu_{1}(y)\right] .
$$

The effect of the time correlation of $\left\{y^{k}\right\}$ is to increase the variance of $\hat{\mu}_{1}^{k}(y)$, as $\tilde{\sigma}^{2}(y)$ is greater than the value of the variance expected in the case of independent samples, i.e. $\sigma^{2}(y)=\gamma_{Y}(0)$. It can further be proven [19] that when $\left\{y^{k}\right\}_{k}$ is a Gaussian time series:

$$
\hat{\mu}_{1}^{k}(y) \sim \mathcal{N}\left(\mu_{1}^{k}(y), \frac{\tilde{\sigma}^{2}(y)}{k}\right)
$$

which constitutes a modified Central Limit Theorem law applicable to a correlated normally distributed time series. The convergence result does hold when $\left\{y^{k}\right\}$ is non-Gaussian while the convergence in distribution to a normal law is subject to other conditions beyond the scope of the present work. The most important result for the following is the value of the variance provided by Eq. (32), not the distribution of $\hat{\mu}_{1}^{k}(y)$. Within the framework of this present study $\hat{\mu}_{1}^{k}(y) \sim \mathcal{N}\left(\mu_{1}^{k}(y), \frac{\tilde{\sigma}^{2}(y)}{k}\right)$ has systematically been observed. A non-normal distribution limit can, however, not be excluded in all generality. Eq. (32) thus allows the calculation of the convergence error, defined by Eq. (17), for $\hat{\mu}_{1}^{k}(y)$ so long as its autocorrelation function $\gamma_{Y}$ is known. During the course of the simulation, at step $k$, Eq. (32) can be evaluated with the statistical estimator (i.e. the sampled value) of the spectrum denoted by $\gamma_{Y}^{k}\left(k^{\prime \prime \prime}\right)$ to differentiate it from the true spectrum $\gamma_{Y}\left(k^{\prime \prime \prime}\right)$ following:

$$
\tilde{\sigma}^{2}(y)=\hat{\gamma}_{Y}^{k}(0)+2 \sum_{k^{\prime \prime \prime}=1}^{K} \hat{\gamma}_{Y}^{k}\left(k^{\prime \prime \prime}\right)
$$




\subsection{Variance of sampled velocity}

In this section, an expression for the variance of $\hat{\mu}_{1}^{k}(\mathbf{y})$ is derived where $\mathbf{y}$ is a cell-based variable that has been obtained via Eq. (4) from a particle-based variable. To distinguish results in this section from those in the previous, y will denoted by $V$ which could for instance designate a velocity component such as $V_{x}$. The estimator $\hat{\mu}_{1}^{k}(V)$ of the mean $\mu_{1}^{k}(V)$ of $V$ is obtained with:

$$
\hat{\mu}_{1}^{k}(V)=\frac{\sum_{k^{\prime}=1}^{k} \sum_{j=1}^{N^{k^{\prime}}} V_{j}^{k^{\prime}}}{\sum_{k^{\prime}=1}^{k} N^{k^{\prime}}}=\frac{\hat{\mu}_{1}^{k}(\Sigma V)}{\hat{\mu}_{1}^{k}(N)},
$$

which is the ratio of two mean estimators where random variable $\Sigma V$ is defined as $\Sigma V^{k} \triangleq \sum_{j=1}^{N^{k}} V_{j}^{k}$. It cannot be put into the form of a single sum of a random variable so as to make amenable to the Central Limit Theorem. That is, there exists no readily obtainable random variable $\Xi$ in the simulation such that

$$
\hat{\mu}_{1}^{k}(V) \equiv \frac{1}{k} \sum_{k^{\prime}=1}^{k} \xi^{k^{\prime}} .
$$

Variable $\Sigma V$ is defined at time step $k$ as $\Sigma V^{k}=V_{1}^{k}+V_{2}^{k}+\ldots+V_{N_{k}-1}^{k}+V_{N_{k}}^{k}$. It is a random sum of random variables and thus inherently depends on the two random variables $N^{k}$ and $V^{k}$ (assuming that $V_{i}^{k}$ are identically distributed). It can readily be shown that

$$
\mathbb{E}[\Sigma V]=\mathbb{E}[N] \mathbb{E}[V]
$$

and

$$
\operatorname{Var}[\Sigma V]=\mathbb{E}\left[\Sigma V^{2}\right]-\mathbb{E}^{2}[\Sigma V]=\mathbb{E}[N] \operatorname{Var}[V]+\operatorname{Var}[N] \mathbb{E}^{2}[V],
$$

while the proof of a Central Limit Theorem for $\Sigma V$, i.e. $\frac{\hat{\mu}_{1}^{k}(\Sigma V)-\mathbb{E}[\Sigma V]}{\sqrt{k \operatorname{Var}[\Sigma V]}} \stackrel{\mathcal{L}}{\rightarrow} \mathcal{N}(0,1)$, can be found in [21]. As previously discussed, $\hat{\mu}_{1}^{k}(\Sigma V) \sim \mathcal{N}\left(\mu_{1}^{k}(\Sigma V), \frac{\tilde{\sigma}^{2, k}(\Sigma V)}{k}\right)$ and $\hat{\mu}_{1}^{k}(N) \sim \mathcal{N}\left(\mu_{1}^{k}(N), \frac{\tilde{\sigma}^{2, k}(N)}{k}\right)$ so that Eq. (36) indicates that $\hat{\mu}_{1}^{k}(V)$ is equal to the ratio of two normal distributions. Random variables $\Sigma V$ and $N$ are, however, correlated with one another much like each is time-correlated with itself. This time-dependent cross-correlation can be estimated as:

$$
\operatorname{Cov}\left(\hat{\mu}_{1}^{k}(\Sigma V), \hat{\mu}_{1}^{k}(N)\right)=\frac{1}{k^{2}} \sum_{k^{\prime}=1}^{k} \sum_{k^{\prime \prime}=1}^{k} \operatorname{Cov}\left(\Sigma V^{k^{\prime}}, N^{k^{\prime \prime}}\right),
$$

A simplified expression can be obtained for the covariance of $\Sigma V^{k^{\prime}}$ and $N^{k^{\prime}}$ at the same time step $k^{\prime}$ as follows

$$
\begin{aligned}
\operatorname{Cov}\left(\Sigma V_{x}^{k^{\prime}}, N^{k^{\prime}}\right) & =\mathbb{E}\left[\Sigma V_{x}^{k^{\prime}} N^{k^{\prime}}\right]-\mathbb{E}\left[\Sigma V_{x}^{k^{\prime}}\right] \mathbb{E}\left[N^{k^{\prime}}\right]= \\
& \mathbb{E}\left[\left(V_{x, 1}^{k^{\prime}}+\ldots+V_{x, N^{k^{\prime}}}^{k^{\prime}}\right) N^{k^{\prime}}\right]-\mathbb{E}\left[\Sigma V^{k^{\prime}}\right] \mathbb{E}\left[N^{k^{\prime}}\right] \approx \mathbb{E}\left[V_{x}^{k^{\prime}} N^{k^{\prime}} N^{k^{\prime}}\right]-\mathbb{E}\left[\Sigma V^{k^{\prime}}\right] \mathbb{E}\left[N^{k^{\prime}}\right]
\end{aligned}
$$

for a large enough number of particles. Assuming that $V_{x}^{k^{\prime}}$ and $N^{k^{\prime}}$ are uncorrelated, $\mathbb{E}\left[V^{k^{\prime}} N^{k^{\prime}} N^{k^{\prime}}\right]=$ $\mathbb{E}\left[N^{k^{\prime}, 2}\right] \mathbb{E}\left[V_{x}^{k^{\prime}}\right]$ so that

$$
\operatorname{Cov}\left(\Sigma V_{x}^{k^{\prime}}, N^{k^{\prime}}\right)=\mathbb{E}\left[V_{x}^{k^{\prime}}\right] \operatorname{Var}\left[N^{k^{\prime}}\right]
$$

and because $N^{k}$ follows a Poisson distribution, $\operatorname{Var}\left[N^{k^{\prime}}\right]=\mathbb{E}\left[N^{k^{\prime}}\right]$ :

$$
\operatorname{Cov}\left(\Sigma V_{x}^{k^{\prime}}, N^{k^{\prime}}\right)=\mathbb{E}\left[V_{x}^{k^{\prime}}\right] \mathbb{E}\left[N^{k^{\prime}}\right]=\mathbb{E}\left[\Sigma V_{x}^{k^{\prime}}\right]
$$


by Eq. (38).

Following the same approach as for Eq. (32), the double sum of Eq. (40) can be simplified so as to yield:

$\operatorname{Cov}\left(\hat{\mu}_{1}^{k}(\Sigma V), \hat{\mu}_{1}^{k}(N)\right) \approx \frac{\gamma_{\Sigma V, N}(0)}{k}+\frac{2}{k} \sum_{\substack{k^{\prime \prime \prime}=-K \\ k^{\prime \prime \prime} \neq 0}}^{K} \gamma_{\Sigma V, N}\left(k^{\prime \prime \prime}\right)=\frac{\sigma^{2}(\Sigma V, N)}{k}+\frac{2}{k} \sum_{\substack{k^{\prime \prime \prime}=-K \\ k^{\prime \prime \prime} \neq 0}}^{K} \gamma_{\Sigma V, N}\left(k^{\prime \prime \prime}\right) \triangleq \frac{\tilde{\sigma}^{2}(\Sigma V, N)}{k}$,

thereby defining $\tilde{\sigma}^{2}(\Sigma V, N)$, where

$$
\gamma_{\Sigma V, N}\left(k^{\prime \prime \prime}\right) \triangleq \operatorname{Cov}\left(\Sigma V^{k^{\prime}}, N^{k^{\prime}+k^{\prime \prime \prime}}\right), \forall k^{\prime}
$$

as $\left\{\Sigma V^{k}\right\}$ and $\left\{N^{k}\right\}$ are stationary processes and altering as follows: $\sigma(\Sigma V, N)=\operatorname{Cov}\left(\Sigma V^{k}, N^{k}\right)$ the notation of the covariance to make it more consistent. Having determined the correlation $\operatorname{Cov}\left(\hat{\mu}_{1}^{k}(\Sigma V), \hat{\mu}_{1}^{k}(N)\right)$ and the normal distribution functions of $\hat{\mu}_{1}^{k}(\Sigma V)$ and $\hat{\mu}_{1}^{k}(N)$ being known, the exact distribution function of their ratio, as defined by Eq. (36), can be determined following the approach presented in [22]. The exact distribution function of the ratio could then, in principle, be used to calculate its variance. The analytical expression of the distribution function presented in [22] is, however, so complicated that the calculation of its variance, even numerically, is difficult. An approximation of the variance of the ratio can, however, be obtained with the delta method [23] as follows:

$$
\tilde{\sigma}^{2}(V) \triangleq k \operatorname{Var}\left[\frac{\mu_{1}^{k}(\Sigma V)}{\mu_{1}^{k}(N)}\right] \approx \frac{\mu_{1}^{2}(\Sigma V)}{\mu_{1}^{4}(N)} \tilde{\sigma}^{2}(N)+\frac{\tilde{\sigma}^{2}(\Sigma V)}{\mu_{1}^{2}(N)}-\frac{2 \mu_{1}(\Sigma V)}{\mu_{1}^{3}(N)} \tilde{\sigma}^{2}(\Sigma V, N)
$$

Eq. (46) thus provides an expression for the variance of the sampled mean velocity $\hat{\mu}_{1}^{k}(V)$ which, by the definition of Eq. (17), is also the convergence error $\tilde{\varepsilon}^{k}\left[\mu_{1}(V)\right]$. It can be evaluated during the course of the simulation by using the statistical estimators that are sampled (designated, following our nomenclature, by $\left.\widehat{(\cdot)}^{k}\right)$ :

$$
\tilde{\sigma}^{2}(V) \approx \frac{\hat{\mu}_{1}^{k, 2}(\Sigma V)}{\hat{\mu}_{k, 1}^{4}(N)}{\widehat{\tilde{\sigma}^{2}}}^{k}(N)+\frac{{\widehat{\tilde{\sigma}^{2}}}^{k}(\Sigma V)}{\hat{\mu}_{k, 1}^{2}(N)}-\frac{2 \hat{\mu}_{1}^{k}(\Sigma V)}{\hat{\mu}_{1}^{k, 3}(N)}{\widehat{\tilde{\sigma}^{2}}}^{k}(\Sigma V, N)
$$

In Eq. (47), ${\widehat{\tilde{\sigma}^{2}}}^{k}(N)$ and ${\widehat{\tilde{\sigma}^{2}}}^{k}(\Sigma V)$ are both evaluated with Eq. (32) respectively applied to $N$ and $\Sigma V$ following

$$
{\widehat{\tilde{\sigma}^{2}}}^{k}(N)=\hat{\gamma}_{N}^{k}(0)+2 \sum_{k^{\prime \prime \prime}=1}^{K} \hat{\gamma}_{N}^{k}\left(k^{\prime \prime \prime}\right)
$$

and

$$
{\widehat{\tilde{\sigma}^{2}}}^{k}(\Sigma V)=\hat{\gamma}_{\Sigma V}^{k}(0)+2 \sum_{k^{\prime \prime \prime}=1}^{K} \hat{\gamma}_{\Sigma V}^{k}\left(k^{\prime \prime \prime}\right)
$$

$\widehat{\tilde{\sigma}}^{k}(\Sigma V, N)$ is determined via Eq. (44) according to

$$
\widehat{\tilde{\sigma}}^{k}(\Sigma V, N)=\hat{\gamma}_{\Sigma V, N}^{k}(0)+2 \sum_{\substack{k^{\prime \prime \prime}=-K \\ k^{\prime \prime \prime} \neq 0}}^{K} \hat{\gamma}_{\Sigma V, N}^{k}\left(k^{\prime \prime \prime}\right)
$$

The determination of the convergence error during the course of the simulation for $\mu_{1}(V), \tilde{\varepsilon}^{k}\left[\mu_{1}(V)\right]$, thus requires the sampling of the autocorrelation function of $N$ and $\Sigma V$, i.e. $\hat{\gamma}_{N}^{k}\left(k^{\prime \prime \prime}\right)$ and $\hat{\gamma}_{\Sigma V}^{k}\left(k^{\prime \prime \prime}\right)$ respectively, and the correlation function of $N$ and $\Sigma V, \hat{\gamma}_{\Sigma V, N}^{k}\left(k^{\prime \prime \prime}\right)$.

The value of $\tilde{\sigma}^{2}(V)$ that could be theoretically obtained if samples did not exhibit time correlation is designated by $\tilde{\sigma}_{0}^{2}(V)$. In the absence of time correlation, $\gamma_{Y}(k)=0, \forall k \geq 1$ so that by Eq. (32), 
$\tilde{\sigma}(N)=\sigma(N), \tilde{\sigma}(\Sigma V)=\sigma(\Sigma V)$ while Eq. (44) yields $\tilde{\sigma}(\Sigma V, N)=\sigma(\Sigma V, N)$. From the approximation of Eq. (43), $\sigma(\Sigma V, N) \approx \mathbb{E}\left[\Sigma V^{k}\right]=\mu_{1}^{k}(\Sigma V)$. Putting everything together, we obtain

$$
\tilde{\sigma}_{0}^{2}(V)=\frac{\mu_{1}^{2}(\Sigma V)}{\mu_{1}^{3}(N)}+\frac{\left[\mu_{1}^{k}(N)+\sigma^{2}(N) \mu_{1}^{2}(V)\right]}{\mu_{1}^{2}(N)}-\frac{2 \mu_{1}^{2}(\Sigma V)}{\mu_{1}^{3}(N)}=\mu_{1}(N) \sigma^{2}(V)
$$

using the expressions of Eqs. (38) and (39) for $\mu_{1}(\Sigma V)$ and $\sigma(\Sigma V)$. As $\tilde{\sigma}^{2}(V)<\tilde{\sigma}_{0}^{2}(V)$, the latter provides a convenient normalization of the convergence error that will later be used for plots.

\section{An approximate correlation determination method}

The approach outlined in the previous section allows the determination of the convergence error for the sampled mean of $y^{k}$ in each cell of the simulation so long as its autocorrelation function $\gamma_{Y}$ is known. In the case of any particle based variable $V$, knowledge of the correlation function between $V$ and $N$ is further required. Because of the amount of information contained in the spectra as well as the fundamental nature of the extended Central Limit Theorem presented, the method is expected to produce an excellent prediction of the convergence error for all DSMC simulations. The accuracy of the prediction will indeed be later illustrated within the framework of the test cases examined in Sections 5 and 6 . The use of the full correlation spectrum to predict the convergence error, however, presents a number of drawbacks which, amongst others, include the following: i) The storage of the correlation spectrum might prove difficult in existing DSMC codes or the implementation of the scheme outlined above might prove to be an excessive effort for most of the practical applications for which DSMC is used. ii) The correlation spectra or convergence properties are not known at the onset of the simulation and a significant number of sampling steps might be required to accurately determine the spectrum (particularly for large $k$ values) iii) Sampling for the correlation spectrum to determine correlation properties amounts to discarding a significant amount of readily obtainable information about the flow which are intuitively known to affect correlation such as the cell size, average velocity or average collision rate. These drawbacks have led to the development of a simplified analytical model to try to predict, without specifically sampling it, the value of the autocorrelation spectrum using basic cell based quantities, (i.e. $\hat{\mu}^{k}(N), \hat{\mu}^{k}(\Sigma V)$ and $\hat{\mu}^{k}\left(\Sigma V^{2}\right)$ ), that are sampled in all DSMC implementations.

\subsection{Number density correlation}

\subsubsection{Consecutive time step correlation}

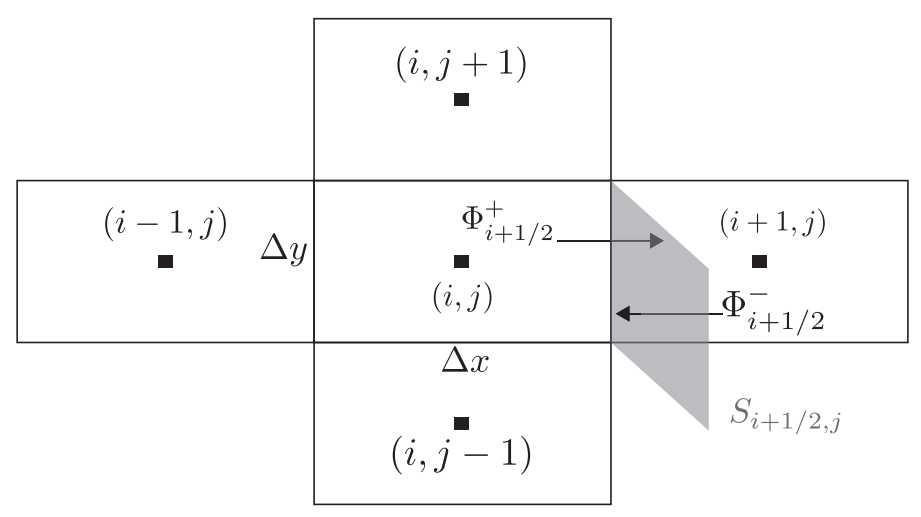

Figure 1: View of cell $(i, j)$ of volume $V_{i, j}$ and fluxes at the $(i+1 / 2, j)$ interface. 
We consider cell $(i, j)$ of the axisymmetric two-dimensional Cartesian mesh depicted in Fig. 1. The number of particles at time step $k$ in cell $(i, j)$ is denoted by $N_{i j}^{k}$. We assume that the flow is equilibrium and that no gradients of macroscopic variables (number density, bulk velocity and temperature) exist in the flow. The change in the number of particles between step $k$ and $k+1$ is due to particles leaving or entering from adjacent cells. The fraction of particles crossing more than one cell per time step is small per DSMC requirement so that it can be neglected. We denote, for instance, the total number of particles entering cell $(i, j)$ from cell $(i-1, j)$ by $\Delta N_{(i-1, j) \rightarrow(i, j)}^{k}>0$ and that leaving $i$ for cell $i+1$ by $\Delta N_{(i, j) \rightarrow(i+1, j)}^{k}>0$. By considering all neighboring cells, the number of particles at step $k+1$ can be found to be:

$$
\begin{aligned}
N_{i j}^{k+1}=N_{i j}^{k}+\Delta N_{(i+1, j) \rightarrow(i, j)}^{k} & +\Delta N_{(i-1, j) \rightarrow(i, j)}^{k}+\Delta N_{(i, j+1) \rightarrow(i, j)}^{k}+\Delta N_{(i, j-1) \rightarrow(i, j)}^{k} \\
& -\Delta N_{(i, j) \rightarrow(i-1, j)}^{k}-\Delta N_{(i, j) \rightarrow(i+1, j)}^{k}-\Delta N_{(i, j) \rightarrow(i, j+1)}^{k}-\Delta N_{(i, j) \rightarrow(i, j-1)}^{k}
\end{aligned}
$$

so that

$$
\begin{array}{r}
\mathbb{E}\left[N_{i j}^{k+1} N_{i j}^{k}\right]-\mathbb{E}\left[N_{i j}^{k, 2}\right]=\mathbb{E}\left[\Delta N_{(i+1, j) \rightarrow(i, j)}^{k} N_{i j}^{k}\right]+\mathbb{E}\left[\Delta N_{(i-1, j) \rightarrow(i, j)}^{k} N_{i j}^{k}\right]+\mathbb{E}\left[\Delta N_{(i, j+1) \rightarrow(i, j)}^{k} N_{i j}^{k}\right] \\
+\mathbb{E}\left[\Delta N_{(i, j-1) \rightarrow(i, j)}^{k} N_{i j}^{k}\right]-\mathbb{E}\left[\Delta N_{(i, j) \rightarrow(i-1, j)}^{k} N_{i j}^{k}\right]-\mathbb{E}\left[\Delta N_{(i, j) \rightarrow(i+1, j)}^{k} N_{i j}^{k}\right]-\mathbb{E}\left[\Delta N_{(i, j) \rightarrow(i, j+1)}^{k} N_{i j}^{k}\right] \\
-\mathbb{E}\left[\Delta N_{(i, j) \rightarrow(i, j-1)}^{k} N_{i j}^{k}\right] .
\end{array}
$$

It can readily be shown by numerical experimentation [2] that the number of outgoing particles (i.e. all $\Delta N_{(i, j) \rightarrow\left(i^{\prime}, j^{\prime}\right)}^{k}$ terms) follows a Poisson distribution and that there exists a constant $C_{(i, j) \rightarrow\left(i^{\prime}, j^{\prime}\right)}>0$ such that

$$
\mathbb{E}\left[\Delta N_{(i, j) \rightarrow\left(i^{\prime}, j^{\prime}\right)}^{k}\right]=C_{(i, j) \rightarrow\left(i^{\prime}, j^{\prime}\right)} \mathbb{E}\left[N_{i j}^{k}\right]
$$

As $N_{i j}^{k}$ also follows a Poisson distribution, a natural approximation is to assume that:

$$
\Delta N_{(i, j) \rightarrow\left(i^{\prime}, j^{\prime}\right)}^{k}=C_{(i, j) \rightarrow\left(i^{\prime}, j^{\prime}\right)} N_{i j}^{k}
$$

A full discussion of the validity of this approximation is outside the scope of this article. A brief comparison is made of the flux obtained with and without this approximation for a one dimensional cell in equilibrium in the Appendix. The value of the proportionality constant $C_{(i, j) \rightarrow\left(i^{\prime}, j^{\prime}\right)}$ can readily be determined in the equilibrium case by recognizing that $\mathbb{E}\left[\Delta N_{(i, j) \rightarrow\left(i^{\prime}, j^{\prime}\right)}^{k}\right]$ can be obtained from the moments of the Maxwellian distribution $f_{i, j}\left(v_{x}, v_{y}, v_{z}\right)$, which we assume exists inside cell $(i, j)$ :

$$
f_{i, j}(\vec{v})=n_{i, j}\left(\frac{m}{2 \pi k_{B} T_{i, j}}\right)^{3 / 2} \exp \left(-\frac{\left(\vec{v}-\vec{u}_{i, j}\right)^{2}}{2 k_{B} T_{i, j}}\right)
$$

where $\vec{u}_{i, j}=\left(u_{x, i, j}, u_{y, i, j}, u_{z, i, j}=0\right), T_{i, j}$ and $n_{i, j}$ are, respectively, the bulk velocity, temperature and number density of cell $(i, j)$. If one, for instance, considers fluxes between cells $(i, j)$ and $(i+1, j)$, the flux of physical particles (number of particles per unit time and area) $\Phi_{i+1 / 2}^{+}(n)$ going from cell $(i, j)$ to $(i+1, j)$ is given by the following

$$
\Phi_{i+1 / 2}^{+}(n)=\int_{-\infty}^{+\infty} d v_{z} \int_{-\infty}^{+\infty} d v_{y} \int_{0}^{+\infty} d v_{x} v_{x} f_{i, j}(\vec{v})=n_{i, j} u_{x, i, j}^{+},
$$

while the flux of particles in the other direction, i.e. from cell $(i+1, j)$ to $(i, j)$ is:

$$
\Phi_{i+1 / 2}^{-}(n)=\int_{-\infty}^{+\infty} d v_{z} \int_{-\infty}^{+\infty} d v_{y} \int_{-\infty}^{0} d v_{x} v_{x} f_{i+1, j}(\vec{v})=n_{i+1, j} u_{x, i+1, j}^{-} .
$$


Since we assume that cells $(i+1, j)$ and $(i, j)$ have the same number density $n_{i, j}$ and distribution function $f_{i, j}$, the integrals can be evaluated analytically [24] to yield

$$
u_{x, i, j}^{ \pm}\left(s_{x}\right) \triangleq \frac{1}{4}\left(\frac{8 k_{B} T_{i, j}}{m}\right)^{1 / 2}\left[\exp \left(-s_{x}^{2}\right)+\sqrt{\pi} s_{x}\left(\operatorname{erf}\left(s_{x}\right) \pm 1\right)\right]
$$

with $s_{x} \triangleq u_{x, i, j} \sqrt{\frac{m}{2 k_{B} T_{i, j}}}$. The expectation of the number of computational particles crossing the $S_{i+1 / 2, j}$ interface during $\Delta t$ can be obtained from the fluxes of Eqs. (57) and (58) by noting that

$$
\begin{gathered}
n_{i, j}=\frac{W_{p} \mathbb{E}\left[N_{i j}^{k}\right]}{V_{i, j}}, \\
\mathbb{E}\left[\Delta N_{(i, j) \rightarrow(i+1, j)}^{k}\right]=\frac{\Delta t S_{i+1 / 2, j}}{W_{p}} \Phi_{i+1 / 2}^{+}(n)=\frac{\Delta t S_{i+1 / 2, j} u_{x, i, j}^{+}}{V_{i, j}} \mathbb{E}\left[N_{i j}^{k}\right] .
\end{gathered}
$$

Comparing Eqs. (61) and (54), one obtains

$$
C_{(i, j) \rightarrow(i+1, j)}=\Delta t \frac{S_{i+1 / 2, j}}{V_{i, j}} u_{x, i, j}^{+}=\frac{u_{x, i, j}^{+} \Delta t}{\Delta x},
$$

by noting that, in an axisymmetric geometry, $V_{i, j} \approx 2 \pi y_{i, j} \Delta y \Delta x$ and $S_{i+1 / 2, j}=2 \pi y_{i, j} \Delta y$, while in a twodimensional $V_{i, j}=\Delta x \Delta y \Delta z$ and $S_{i+1 / 2, j}=\Delta y \Delta z$ with $\Delta z=1$. By considering the flux of particles in the opposite direction from cell $(i+1, j)$ to $(i, j)$, one similarly obtains:

$$
C_{(i+1, j) \rightarrow(i, j)}=\frac{u_{x, i, j}^{-} \Delta t}{\Delta x}
$$

The value of the fluxes in the $y$ direction are also obtained in a similar fashion by replacing $u_{x, i, j}^{ \pm}$by

$$
u_{y, i, j}^{ \pm}=u_{x, i, j}^{ \pm}\left(s_{y}\right)
$$

with $s_{y} \triangleq u_{y, i, j} \sqrt{\frac{m}{2 k_{B} T_{i, j}}}$, so that one, for instance, has:

$$
C_{(i, j) \rightarrow(i, j+1)}=\frac{u_{y, i, j}^{+} \Delta t}{\Delta y}
$$

Returning to Eq. (53) and using Eq. (55), written from the standpoint of cell $\left(i^{\prime}, j^{\prime}\right)$, all terms involving incoming fluxes of particles can be rewritten as:

$$
\begin{aligned}
\mathbb{E}\left[\Delta N_{\left(i^{\prime}, j^{\prime}\right) \rightarrow(i, j)}^{k} N_{i j}^{k}\right]=\mathbb{E}\left[C_{\left(i^{\prime}, j^{\prime}\right) \rightarrow(i, j)} N_{\left(i^{\prime}, j^{\prime}\right) \rightarrow(i, j)}^{k} N_{i j}^{k}\right]=C_{\left(i^{\prime}, j^{\prime}\right) \rightarrow(i, j)} \mathbb{E}\left[N_{i^{\prime} j^{\prime}}^{k}\right] \mathbb{E}\left[N_{i, j}^{k}\right] \\
=C_{\left(i^{\prime}, j^{\prime}\right) \rightarrow(i, j)} \mathbb{E}^{2}\left[N_{i j}^{k}\right]
\end{aligned}
$$

by assuming that $N_{i, j}^{k}$ and $N_{i^{\prime}, j^{\prime}}^{k}$ are independent random variables (and that $\mathbb{E}\left[N_{i^{\prime}, j^{\prime}}^{k}\right]=\mathbb{E}\left[N_{i, j}^{k}\right]$ for the last equality). The terms of Eq. (53) containing outgoing particle fluxes can be rewritten, using Eq. (55), as

$$
\mathbb{E}\left[\Delta N_{(i, j) \rightarrow\left(i^{\prime}, j^{\prime}\right)}^{k} N_{i j}^{k}\right]=C_{(i, j) \rightarrow\left(i^{\prime}, j^{\prime}\right)} \mathbb{E}\left[N_{i j}^{k, 2}\right]
$$

so that, using Eq. (66), Eq. (53) becomes

$$
\begin{array}{r}
\mathbb{E}\left[N_{i j}^{k+1} N_{i j}^{k}\right]-\mathbb{E}\left[N_{i j}^{k, 2}\right]=C_{(i+1, j) \rightarrow(i, j)} \mathbb{E}^{2}\left[N_{i j}^{k}\right]+C_{(i-1, j) \rightarrow(i, j)} \mathbb{E}^{2}\left[N_{i j}^{k}\right]+C_{(i, j+1) \rightarrow(i, j)} \mathbb{E}^{2}\left[N_{i j}^{k}\right] \\
+C_{(i, j-1) \rightarrow(i, j)} \mathbb{E}^{2}\left[N_{i j}^{k}\right]-C_{(i, j) \rightarrow(i+1, j)} \mathbb{E}\left[N_{i j}^{k, 2}\right]-C_{(i, j) \rightarrow(i-1, j)} \mathbb{E}\left[N_{i j}^{k, 2}\right] \\
-C_{(i, j) \rightarrow(i, j+1)} \mathbb{E}\left[N_{i j}^{k, 2}\right]-C_{(i, j) \rightarrow(i, j-1)} \mathbb{E}\left[N_{i j}^{k, 2}\right]
\end{array}
$$


From the homogeneity assumption, $C_{(i \pm 1, j) \rightarrow(i, j)}=C_{(i, j) \rightarrow(i \mp 1, j)}$ and $C_{(i, j \pm 1) \rightarrow(i, j)}=C_{(i, j) \rightarrow(i, j \mp 1)}$, so that Eq. (68) becomes

$$
\begin{array}{r}
\mathbb{E}\left[N_{i j}^{k+1} N_{i j}^{k}\right]-\mathbb{E}^{2}\left[N_{i j}^{k}\right]=\operatorname{Var}\left[N_{i, j}^{k}\right]-C_{(i, j) \rightarrow(i+1, j)} \operatorname{Var}\left[N_{i j}^{k}\right]-C_{(i, j) \rightarrow(i-1, j)} \operatorname{Var}\left[N_{i j}^{k}\right] \\
-C_{(i, j) \rightarrow(i, j+1)} \operatorname{Var}\left[N_{i j}^{k}\right]-C_{(i, j) \rightarrow(i, j-1)} \operatorname{Var}\left[N_{i j}^{k}\right]
\end{array}
$$

since $\operatorname{Var}\left[N_{i j}^{k}\right]=\mathbb{E}\left[N_{i j}^{k, 2}\right]-\mathbb{E}^{2}\left[N_{i j}^{k}\right]$. Finally, substituting for the flux terms, and using the definition of the autocorrelation function $\rho$ of Eq. (28):

$$
\rho_{N_{i j}}(k=1)=1-\frac{\underline{\mathrm{u}}_{x, i, j} \Delta t}{\Delta x}-\frac{\underline{\mathrm{u}}_{y, i, j} \Delta t}{\Delta y},
$$

with $\underline{\mathrm{u}}_{x, i, j} \triangleq u_{x, i, j}^{+}+u_{x, i, j}^{-}$and $\underline{\underline{u}}_{y, i, j} \triangleq u_{y, i, j}^{+}+u_{y, i, j}^{-}, u^{ \pm}$having been defined in Eq. (59). By analogy with finite difference methods, we define a cell Courant-like number, which we denote by $\mathcal{C}$, as

$$
\mathcal{C}=\frac{\underline{\mathrm{u}}_{x, i, j} \Delta t}{\Delta x}+\frac{\underline{\mathrm{u}}_{y, i, j} \Delta t}{\Delta y}
$$

so that Eq. (70) becomes:

$$
\rho_{N}(k=1)=1-\mathcal{C} .
$$

Eq. (72) thus provides an approximate expression for the first value of the autocorrelation spectrum. The accuracy of this estimation will be examined in Sections 5 and 6 .

\subsubsection{Spectrum approximation}

The entire correlation spectrum, i.e. $\rho(k), k \geq 1$ can be obtained from the value of the first time correlation $\rho(1)$. We claim that

$$
\rho_{N}(k)=(1-\mathcal{C})^{k}, \quad k \geq 0
$$

which we proceed to prove by induction in the following.

Proof: Equation (73) is indeed valid for $k=1$ as shown above. We now assume that

$$
\rho_{N}\left(k^{\prime}\right)=(1-\mathcal{C})^{k^{\prime}}
$$

and examine the value of $\rho_{N}\left(k^{\prime}+1\right)$. By definition:

$$
\mathbb{E}\left[N_{i j}^{k+k^{\prime}+1} N_{i j}^{k}\right]=\mathbb{E}\left[\left(N_{i j}^{k+k^{\prime}}+\Delta N_{i j}^{k+k^{\prime}}\right) N_{i j}^{k}\right]=\mathbb{E}\left[N_{i j}^{k+k^{\prime}} N_{i j}^{k}\right]+\mathbb{E}\left[N_{i j}^{k+k^{\prime}} \Delta N_{\rightarrow i j}^{k}\right]-\mathbb{E}\left[N_{i j}^{k+k^{\prime}} \Delta N_{i j \rightarrow}^{k}\right]
$$

lumping incoming and outgoing fluxes from/to all directions into $\Delta N_{\rightarrow i j}^{k}$ and $\Delta N_{i j \rightarrow}^{k}$ respectively. Using Eq. (74):

$$
\mathbb{E}\left[N_{i j}^{k+k^{\prime}} N_{i j}^{k}\right]=(1-\mathcal{C})^{k^{\prime}} \operatorname{Var}\left[N_{i j}^{k}\right]+\mathbb{E}^{2}\left[N_{i j}^{k}\right],
$$

while the last two terms of Eq. (75) (only considering the $x$ direction and $i+1 / 2$ interface for the sake of conciseness) can be rewritten, following the same approach as in Eq. (70), as:

$$
\mathbb{E}\left[N_{i j}^{k+k^{\prime}} \Delta N_{\rightarrow i j}^{k}\right]-\mathbb{E}\left[N_{i j}^{k+k^{\prime}} \Delta N_{i j \rightarrow}^{k}\right]=\frac{\underline{u}_{x, i, j}^{-} \Delta t}{\Delta x} \mathbb{E}\left[N_{i+1, j}^{k+k^{\prime}} N_{i, j}^{k}\right]-\frac{\underline{u}_{x, i, j}^{+} \Delta t}{\Delta x} \mathbb{E}\left[N_{i, j}^{k+k^{\prime}} N_{i, j}^{k}\right] .
$$

Assuming $N_{i+1, j}^{k+k^{\prime}}$ and $N_{i, j}^{k}$ to be independent, $\mathbb{E}\left[N_{i+1, j}^{k+k^{\prime}} N_{i, j}^{k}\right]=\mathbb{E}\left[N_{i+1, j}^{k+k^{\prime}}\right] \mathbb{E}\left[N_{i, j}^{k}\right]=\mathbb{E}^{2}\left[N_{i, j}^{k}\right]$, while from Eq. (76), $\mathbb{E}\left[N_{i, j}^{k+k^{\prime}} N_{i, j}^{k}\right]=(1-\mathcal{C})^{k^{\prime}} \operatorname{Var}\left[N_{i j}^{k}\right]+\mathbb{E}^{2}\left[N_{i j}^{k}\right]$, Eq. (77) thus becomes:

$$
\mathbb{E}\left[N_{i j}^{k+k^{\prime}} \Delta N_{\rightarrow i j}^{k}\right]-\mathbb{E}\left[N_{i j}^{k+k^{\prime}} \Delta N_{i j \rightarrow}^{k}\right]=\frac{\underline{u}_{x, i, j}^{-} \Delta t}{\Delta x} \mathbb{E}^{2}\left[N_{i, j}^{k}\right]-\frac{\underline{u}_{x, i, j}^{+} \Delta t}{\Delta x}\left\{(1-\mathcal{C})^{k^{\prime}} \operatorname{Var}\left[N_{i j}^{k}\right]+\mathbb{E}^{2}\left[N_{i j}^{k}\right]\right\}
$$


When both directions and all interfaces are considered, Eq. (78) can be found to be equivalent to:

$$
\mathbb{E}\left[N_{i j}^{k+k^{\prime}} \Delta N_{\rightarrow i j}^{k}\right]-\mathbb{E}\left[N_{i j}^{k+k^{\prime}} \Delta N_{i j \rightarrow}^{k}\right]=-\mathcal{C}\left\{(1-\mathcal{C})^{k^{\prime}} \operatorname{Var}\left[N_{i j}^{k}\right]\right\}
$$

Inserting Eqs. (79) and (76) into Eq. (75) finally yields:

$$
\mathbb{E}\left[N_{i j}^{k+k^{\prime}+1} N_{i j}^{k}\right]-\mathbb{E}^{2}\left[N_{i j}^{k}\right]=(1-\mathcal{C})^{k^{\prime}} \operatorname{Var}\left[N_{i j}^{k}\right]-\mathcal{C}\left\{(1-\mathcal{C})^{k^{\prime}} \operatorname{Var}\left[N_{i j}^{k}\right]\right\}
$$

$$
=(1-\mathcal{C})^{k^{\prime}+1} \operatorname{Var}\left[N_{i j}^{k}\right]
$$

and hence

so that by induction

$$
\rho_{N}\left(k^{\prime}+1\right)=(1-\mathcal{C})^{k^{\prime}+1}
$$

$$
\rho_{N}(k)=(1-\mathcal{C})^{k}, \quad k \geq 0
$$

which completes the proof. Because, from Eq. (82), the spectrum follows a geometric series with argument $r=1-\mathcal{C}$, the sum that appears in Eq. (32) can be simplified as follows:

$$
\operatorname{Var}\left[\hat{\mu}_{1}^{k}(N)\right]=\frac{\tilde{\sigma}^{2, k}(N)}{k}=\frac{\sigma^{2}(N)}{k}\left[1+2 \sum_{k^{\prime \prime \prime}=1}^{K} \rho_{N}\left(k^{\prime \prime \prime}\right)\right] \approx \frac{\hat{\mu}_{1}^{k}(N)}{k}\left[\frac{2}{\mathcal{C}}-1\right]
$$

as $(1-\mathcal{C})^{K} \approx 0$. Eq. (83) thus provides an approximate value for the convergence error of each individual cell as a function of the number of samples $k$ and the cell Courant number. The Courant number is calculated via Eq. (71) which only requires estimates of the cell bulk velocity, i.e. $\vec{u}_{i, j}=\left(u_{x, i, j}, u_{y, i, j}\right)$, and temperature $T_{i, j}$. During the sampling period, the most natural estimates for these is are sampled velocity and temperature which are available in all implementations of the DSMC method.

\subsection{Velocity correlation}

\subsubsection{Consecutive time step correlation}

We are interested in obtaining an approximate expression for Eq. (46) that does not require the calculation of any correlation spectrum. Having already obtained an expression for the correlation of $N$, we now turn to that of $\Sigma V=\Sigma V_{x}$. As will later become apparent, the exact same approach can be utilized for the $y$ or $z$ (for 3D simulations) velocity components. Following the same approach as previously for $N^{k}$, we consider the change of $\Sigma V_{x}$, i.e. the $x$-momentum divided by $m$, of cell $(i, j)$ depicted in Fig. 1.

$$
\begin{gathered}
\Sigma V_{x, i, j}^{k+1}=\Sigma V_{x, i, j}^{k}+\Delta \Sigma V_{x,(i+1, j) \rightarrow(i, j)}^{k}+\Delta \Sigma V_{x,(i-1, j) \rightarrow(i, j)}^{k}+\Delta \Sigma V_{x,(i, j+1) \rightarrow(i, j)}^{k}+\Delta \Sigma V_{x,(i, j-1) \rightarrow(i, j)}^{k} \\
-\Delta \Sigma V_{x,(i, j) \rightarrow(i+1, j)}^{k}-\Delta \Sigma V_{x,(i, j) \rightarrow(i-1, j)}^{k}-\Delta \Sigma V_{x,(i, j) \rightarrow(i, j+1)}^{k}-\Delta \Sigma V_{x,(i, j) \rightarrow(i, j-1)}^{k} \\
+\Delta \Sigma V_{x, i, j \mathrm{coll}}^{k}
\end{gathered}
$$

where terms $\Delta \Sigma V_{x,\left(i^{\prime}, j^{\prime}\right) \rightarrow(i, j)}^{k}$ and $\Delta \Sigma V_{x,(i, j) \rightarrow\left(i^{\prime}, j^{\prime}\right)}^{k}$ represent, respectively, incoming and outgoing fluxes of particles x-velocity from/to adjacent cells. The effect of collisions during $\Delta t$ on the sum of velocities in the cell is contained in the $\Delta \Sigma V_{x, i, j, \text { coll }}^{k}$ term. The first time correlation of $\Sigma V_{x, i, j}^{k}$ is thus obtained from Eq. (84) as:

$$
\begin{array}{r}
\mathbb{E}\left[\Sigma V_{x, i, j}^{k+1} \Sigma V_{x, i, j}^{k}\right]-\mathbb{E}\left[\Sigma V_{x, i, j}^{k, 2}\right]=\mathbb{E}\left[\Delta \Sigma V_{x,(i+1, j) \rightarrow(i, j)}^{k} \Sigma V_{x, i, j}^{k}\right]+\mathbb{E}\left[\Delta \Sigma V_{x,(i-1, j) \rightarrow(i, j)}^{k} \Sigma V_{x, i, j}^{k}\right] \\
+\mathbb{E}\left[\Delta \Sigma V_{x,(i, j+1) \rightarrow(i, j)}^{k} \Sigma V_{x, i, j}^{k}\right]+\mathbb{E}\left[\Delta \Sigma V_{x,(i, j-1) \rightarrow(i, j)}^{k} \Sigma V_{x, i, j}^{k}\right]-\mathbb{E}\left[\Delta \Sigma V_{x,(i, j) \rightarrow(i+1, j)}^{k} \Sigma V_{x, i, j}^{k}\right] \\
-\mathbb{E}\left[\Delta \Sigma V_{x,(i, j) \rightarrow(i-1, j)}^{k} \Sigma V_{x, i, j}^{k}\right]-\mathbb{E}\left[\Delta \Sigma V_{x,(i, j) \rightarrow(i, j+1)}^{k} \Sigma V_{x, i, j}^{k}\right]-\mathbb{E}\left[\Delta \Sigma V_{x,(i, j) \rightarrow(i, j-1)}^{k} \Sigma V_{x, i, j}^{k}\right] \\
+\mathbb{E}\left[\Delta \Sigma V_{x, i, j, \mathrm{coll}}^{k} \Sigma V_{x, i, j}^{k}\right]
\end{array}
$$


As previously, we assume that $\Delta \Sigma V_{x,\left(i^{\prime}, j^{\prime}\right) \rightarrow(i, j)}^{k}$ and $\Sigma V_{x, i, j}^{k}$ are independent, so that all terms involving incoming fluxes on the right hand side of Eq. (85), i.e. $\mathbb{E}\left[\Delta \Sigma V_{x,\left(i^{\prime}, j^{\prime}\right) \rightarrow(i, j)}^{k} \Sigma V_{x, i, j}^{k}\right]$, can be rewritten as $\mathbb{E}\left[\Delta \Sigma V_{x,\left(i^{\prime}, j^{\prime}\right) \rightarrow(i, j)}^{k}\right] \mathbb{E}\left[\Sigma V_{x, i, j}^{k}\right]$. In order to approximate the terms involving outgoing fluxes, i.e. $\mathbb{E}\left[\Delta \Sigma V_{x,(i, j) \rightarrow\left(i^{\prime}, j^{\prime}\right)}^{k} \Sigma V_{x, i, j}^{k}\right]$, we need to express the flux as a function of $\Sigma V_{x, i, j}^{k}$. In the case of the number of particles flux that was studied in the previous section, the number of particles flux, as well as the number of particles in the cell $N^{k}$, both follow Poisson distributions so that one can naturally postulate that $\Delta N_{(i, j) \rightarrow\left(i^{\prime}, j^{\prime}\right)}^{k}=C_{(i, j) \rightarrow\left(i^{\prime}, j^{\prime}\right)} N_{i, j}^{k}$ although we had to assume that $\rho\left(\Delta N_{(i, j) \rightarrow\left(i^{\prime}, j^{\prime}\right)}^{k}, N_{i, j}^{k}\right)=1$. Here, when most particles are such that $v>\sqrt{2 R T}$, as is the case in most high speed flows, such as in the test case of Section 5.1, $\Delta \Sigma V_{x,(i, j) \rightarrow\left(i^{\prime}, j^{\prime}\right)}^{k}$ can very well be approximated by a normal distribution. As $\Sigma V_{x,(i, j)}^{k}$ also follows a normal distribution, a natural approximate expression for $\Delta \Sigma V_{x,(i, j) \rightarrow\left(i^{\prime}, j^{\prime}\right)}^{k}$ is thus

$$
\Delta \Sigma V_{x,(i, j) \rightarrow\left(i^{\prime}, j^{\prime}\right)}^{k}=C_{x,(i, j) \rightarrow\left(i^{\prime}, j^{\prime}\right)} \Sigma V_{x,(i, j)}^{k}
$$

where $C_{x,(i, j) \rightarrow\left(i^{\prime}, j^{\prime}\right)}$ is constant. This assumes complete correlation, i.e. $\rho\left(\Delta \Sigma V_{x,(i, j) \rightarrow\left(i^{\prime}, j^{\prime}\right)}^{k}, \Sigma V_{x, i, j}^{k}\right)=1$, for lack of a better model. This value is, of course, higher than in reality. In view of the satisfactory results obtained with the approximate correlation model in Sections 5 and 6 , this assumption will later be shown to be well-warranted within the context of this simple model. The value of constant $C_{x,(i, j) \rightarrow\left(i^{\prime}, j^{\prime}\right)}$ is obtained by considering the expectation of Eq. (86) and recognizing that exact analytical expressions can be obtained for $\mathbb{E}\left[\Delta \Sigma V_{x,(i, j) \rightarrow\left(i^{\prime}, j^{\prime}\right)}^{k}\right]$ which we proceed to do below. The flux of $x$-momentum divided by the mass $\Phi_{i+1 / 2}^{+}\left(\Sigma V_{x}\right)$ going from cell $(i, j)$ to $(i+1, j)$ is given by the following

$$
\Phi_{i+1 / 2}^{+}\left(\Sigma V_{x}\right)=\int_{-\infty}^{+\infty} d v_{z} \int_{-\infty}^{+\infty} d v_{y} \int_{0}^{+\infty} d v_{x} v_{x}^{2} f_{i, j}(\vec{v})=n_{i, j} \Sigma u_{x x, i, j}^{2,+}
$$

while the flux of velocity sum in the other direction, i.e. from cell $(i+1, j)$ to $(i, j)$ is:

$$
\Phi_{i+1 / 2}^{-}\left(\Sigma V_{x}\right)=\int_{-\infty}^{+\infty} d v_{z} \int_{-\infty}^{+\infty} d v_{y} \int_{-\infty}^{0} d v_{x} v_{x}^{2} f_{i+1, j}(\vec{v})=n_{i, j} \Sigma u_{x x, i, j}^{2,-} .
$$

Assuming that cells $(i+1, j)$ and $(i, j)$ have the same number density and distribution function (given by Eq. (56)), the integrals can be evaluated to yield:

$$
\Sigma u_{x x, i, j}^{2, \pm}\left(s_{x}\right) \triangleq k_{B} T_{i, j}\left[\frac{s_{x}}{\sqrt{\pi}} \exp \left(-s_{x}^{2}\right)+\left(\frac{1}{2}+s_{x}^{2}\right)\left[\operatorname{erf}\left(s_{x}\right) \pm 1\right]\right]
$$

with $s_{x} \triangleq u_{x, i, j} \sqrt{\frac{m}{2 k_{B} T_{i, j}}}$. The fluxes of $\Sigma V_{x}$ in the $y$ direction also have to be considered. The flux of x-momentum divided by the mass $\Phi_{j+1 / 2}^{+}\left(\Sigma V_{x}\right)$ going from cell $(i, j)$ to $(i, j+1)$ is given by the following

$$
\Phi_{j+1 / 2}^{+}\left(\Sigma V_{x}\right)=\int_{-\infty}^{+\infty} d v_{z} \int_{0}^{+\infty} v_{y} d v_{y} \int_{-\infty}^{+\infty} d v_{x} v_{x} f_{i, j}(\vec{v})=n_{i, j} \Sigma u_{x y, i, j}^{2,+}
$$

while that going from cell $(i, j+1)$ to $(i, j)$ is:

$$
\Phi_{j+1 / 2}^{-}\left(\Sigma V_{x}\right)=\int_{-\infty}^{+\infty} d v_{z} \int_{-\infty}^{0} v_{y} d v_{y} \int_{-\infty}^{+\infty} d v_{x} v_{x} f_{i, j}(\vec{v})=n_{i, j} \Sigma u_{x y, i, j}^{2,-}
$$

with

$$
\Sigma u_{x y, i, j}^{2, \pm} \triangleq u_{x, i, j} u_{y, i, j}^{ \pm}
$$


where the $u_{y, i, j}^{ \pm}$terms were previously defined in Eq. (64). All the $C_{x,(i, j) \rightarrow\left(i^{\prime}, j^{\prime}\right)}$ constants of Eq. (86) can thus now be obtained by considering the expectation of that equation. For instance, in the case of $C_{x,(i, j) \rightarrow(i+1, j)}$ :

$$
C_{x,(i, j) \rightarrow(i+1, j)}=\frac{\mathbb{E}\left[\Delta \Sigma V_{x,(i, j) \rightarrow(i+1, j)}^{k}\right]}{\mathbb{E}\left[\Sigma V_{x,(i, j)}^{k}\right]}=\frac{\Delta t S_{i+1 / 2, j}}{W_{p}} \frac{\Phi_{i+1 / 2}^{+}\left(\Sigma V_{x}\right)}{\mathbb{E}\left[N_{i, j}\right] \mathbb{E}\left[V_{x, i, j}\right]}=\frac{\Delta t}{\Delta x} \frac{\Sigma u_{x x, i, j}^{2,+}}{u_{x, i, j}}
$$

using $\mathbb{E}\left[\Delta \Sigma V_{x,(i, j) \rightarrow(i+1, j)}^{k}\right]=\frac{\Delta t S_{i+1 / 2, j} \Phi_{j+1 / 2}^{+}\left(\Sigma V_{x}\right)}{W_{p}}$ and Eqs. (38) and (60). When all the other terms in Eq. (85) are so expressed, and by analogy with Eq. (70), the former becomes:

$$
\begin{array}{r}
\frac{\mathbb{E}\left[\Sigma V_{x, i, j}^{k+1} \Sigma V_{x, i, j}^{k}\right]-\mathbb{E}\left[\Sigma V_{x, i, j}^{k, 2}\right]}{\operatorname{Var}\left[\Sigma V_{x, i, j}\right]}=1-\frac{\Delta t}{\Delta x} \frac{\Sigma u_{x x, i, j}^{2,+}}{u_{x, i, j}}-\frac{\Delta t}{\Delta x} \frac{\Sigma u_{x x, i, j}^{2,-}}{u_{x, i, j}}-\frac{\Delta t}{\Delta y} \frac{\Sigma u_{x y, i, j}^{2,+}}{u_{x, i, j}}-\frac{\Delta t}{\Delta y} \frac{\Sigma u_{x y, i, j}^{2,-}}{u_{x, i, j}} \\
+\frac{\mathbb{E}\left[\Delta \Sigma V_{x, i, j \operatorname{coll}}^{k} \Sigma V_{x, i, j}^{k}\right]}{\operatorname{Var}\left[\Sigma V_{x, i, j}\right]}
\end{array}
$$

We now turn to the collision term on the right hand side of Eq. (94). To simplify our presentation, we isolate the effect of collisions by considering Eq. (85) without flux terms:

$$
\mathbb{E}\left[\Sigma V_{x, i, j}^{k+1} \Sigma V_{x, i, j}^{k}\right]=\mathbb{E}\left[\Sigma V_{x, i, j}^{k, 2}-\Delta \Sigma V_{x, i, j \operatorname{coll}}^{k} \Sigma V_{x, i, j}^{k}+\Delta \Sigma V_{x, i, j \text { coll }}^{\prime k} \Sigma V_{x, i, j}^{k}\right]
$$

The effect of collisions is that a part of the velocity sum, $\Delta \Sigma V_{x, i, j \text { coll }}^{k}$, (the sum of pre-collision velocities of particles that are going to collide) is replaced at the end of the time step by the sum of post-collision velocities $\Delta \Sigma V_{x, i, j \text { coll }}^{\prime}$. Expanding Eq. (95), by assuming that $N_{\text {coll }}^{k}$ particles participate in collisions, and designating by $V_{x, l}^{\prime k}$ the post-collision velocity of particle $l, 1 \leq l \leq N_{\text {coll }}^{k}$, with pre-collision velocity $V_{x, l}$

$$
\begin{array}{r}
\mathbb{E}\left[\Sigma V_{x, i, j}^{k+1} \Sigma V_{x, i, j}^{k}\right]=\mathbb{E}\left[\Sigma V_{x, i, j}^{k, 2}\right]-\mathbb{E}\left[\left(V_{x, 1}^{k}+\ldots+V_{x, N_{\mathrm{coll}, i, j}^{k}}^{k}\right)\left(V_{x, 1}^{k}+\ldots+V_{x, N_{i, j}^{k}}^{k}\right)\right]+ \\
\mathbb{E}\left[\left(V_{x, 1}^{\prime k}+\ldots+V_{x, N_{\mathrm{coll}, i, j}^{\prime}}^{\prime k}\right)\left(V_{x, 1}^{k}+\ldots+V_{x, N_{i, j}^{k}}^{k}\right)\right]
\end{array}
$$

which can be approximated as:

$$
\mathbb{E}\left[\Sigma V_{x, i, j}^{k+1} \Sigma V_{x, i, j}^{k}\right]=\mathbb{E}\left[\Sigma V_{x, i, j}^{k, 2}\right]-\mathbb{E}\left[N_{\mathrm{coll}, i, j}^{k}\right] \mathbb{E}\left[N_{i, j}^{k}\right] \mathbb{E}\left[V_{x, i, j}^{k, 2}\right]+\mathbb{E}\left[N_{\mathrm{coll}, i, j}^{k}\right] \mathbb{E}\left[N_{i, j}^{k}\right] \mathbb{E}\left[V_{x, i, j}^{k} V_{x, i, j}^{\prime k}\right]
$$

The effect of collisions is that post-collision velocities become uncorrelated with pre-collision velocities so that $\mathbb{E}\left[V_{x, i, j}^{k} V_{x, i, j}^{\prime k}\right]=\mathbb{E}\left[V_{x, i, j}^{k}\right] \mathbb{E}\left[V_{x, i, j}^{\prime k}\right] \approx \mathbb{E}^{2}\left[V_{x, i, j}^{k}\right]$ because we assume equilibrium so that, using Eqs. (39) and (97):

$$
\frac{\mathbb{E}\left[\Sigma V_{x, i, j}^{k+1} \Sigma V_{x, i, j}^{k}\right]-\mathbb{E}\left[\Sigma V_{x, i, j}^{k, 2}\right]}{\operatorname{Var}\left[\Sigma V_{x, i, j}\right]}=-\mathbb{E}\left[N_{\mathrm{coll}, i, j}^{k}\right]=-2 \bar{N}_{\mathrm{coll}, i, j}
$$

where $\bar{N}_{\mathrm{coll}, i, j} \approx 2 N_{\mathrm{coll}, i, j}^{k}$ designates the average number of collisions per time step in cell $(i, j)$. Inserting the result of Eq. (98) into Eq. (94), we finally obtain

$$
\rho_{\Sigma V_{x}}(k=1)=1-\mathcal{C}_{\Sigma V_{x}}
$$

by defining the velocity sum Courant number, $\mathcal{C}_{\Sigma V_{x}}$, as follows:

$$
\mathcal{C}_{\Sigma V_{x}}=\frac{\Delta t}{\Delta x} \frac{\Sigma u_{x x, i, j}^{2,+}}{u_{x, i, j}}-\frac{\Delta t}{\Delta x} \frac{\Sigma u_{x x, i, j}^{2,-}}{u_{x, i, j}}-\frac{\Delta t}{\Delta y} \frac{\Sigma u_{x y, i, j}^{2,+}}{u_{x, i, j}}-\frac{\Delta t}{\Delta y} \frac{\Sigma u_{x y, i, j}^{2,-}}{u_{x, i, j}}-2 \bar{N}_{\mathrm{coll}, i, j} .
$$




\subsubsection{Spectrum approximation}

The exact same procedure as for the $N$ autocorrelation spectrum may be used for that of $\Sigma V_{x}$, so that, by analogy with Eq. (83),

$$
\operatorname{Var}\left[\hat{\mu}_{1}^{k}\left(\Sigma V_{x}\right)\right]=\frac{\tilde{\sigma}^{2}\left(\Sigma V_{x}\right)}{k}=\frac{\sigma^{2}\left(\Sigma V_{x}\right)}{k}\left[1+2 \sum_{k^{\prime \prime \prime}=1}^{K} \rho_{\Sigma V_{x}}\left(k^{\prime \prime \prime}\right)\right]=\frac{\sigma^{2}\left(\Sigma V_{x}\right)}{k}\left[\frac{2}{\mathcal{C}_{\Sigma V_{x}}}-1\right]
$$

To finish the formulation of the approximate correlation method, we seek an approximation for the last term of Eq. (46), namely:

$$
\tilde{\sigma}(\Sigma V, N)=\frac{\gamma_{\Sigma V, N}(0)}{k}+\frac{2}{k} \sum_{\substack{k^{\prime \prime \prime}=-K \\ k^{\prime \prime \prime} \neq 0}}^{K} \gamma_{\Sigma V, N}\left(k^{\prime \prime \prime}\right),
$$

We first examine $\gamma_{\Sigma V, N}(1)=\operatorname{Cov}\left(\Sigma V_{x}^{k^{\prime}}, N^{k^{\prime}+1}\right)$ before constructing an approximation of the entire spectrum $\gamma_{\Sigma V, N}(k)=\operatorname{Cov}\left(\Sigma V_{x}^{k^{\prime}}, N^{k^{\prime}+k}\right)$. To keep the notation more concise, the cell subscript indices are dropped in the following, as no spatial variations are considered. The value of the first time correlation, $\gamma_{\Sigma V, N}(k=1)$, noting that $\operatorname{Cov}\left(\Sigma V_{x}^{k^{\prime}}, N^{k^{\prime}}\right)=\mathbb{E}\left[V_{x}^{k^{\prime}}\right] \mathbb{E}\left[N^{k^{\prime}}\right]=\mathbb{E}\left[\Sigma V_{x}^{k^{\prime}}\right]=\gamma_{\Sigma V_{x}, N}(0)$, as obtained in Eq. (43), is given by:

$$
\gamma_{\Sigma V_{x}, N}(k=1)=\operatorname{Cov}\left(\Sigma V_{x}^{k^{\prime}}, N^{k^{\prime}+1}\right) \approx \mathbb{E}\left[N^{k^{\prime}} N^{k^{\prime}+1}\right] \mathbb{E}\left[V_{x}^{k^{\prime}}\right]-\mathbb{E}^{2}\left[N^{k^{\prime}}\right] \mathbb{E}\left[V_{x}^{k^{\prime}}\right] .
$$

Since $\mathbb{E}\left[N^{k^{\prime}} N^{k^{\prime}+1}\right]=\mathbb{E}\left[N^{k^{\prime}}\right] \rho_{N}(1)+\mathbb{E}^{2}\left[N^{k^{\prime}}\right]$ :

$$
\gamma_{\Sigma V_{x}, N}(k=1)=\rho_{N}(1) \gamma_{\Sigma V_{x}, N}(0) .
$$

Using the same approach as in section 4.1.2, it may be proven by induction that

$$
\rho_{\Sigma V_{x}, N}(k)=\rho_{N}(k),
$$

which means that the right hand side of Eq. (102) can be approximated as was done for the spectrum of $N$ in Eq. (83), by further assuming the spectrum to be symmetric with respect to $k$ (an assumption that will later be checked):

$$
\tilde{\sigma}(\Sigma V, N)=\mu_{1}\left(\Sigma V_{x}\right)\left[\frac{2}{\mathcal{C}}-1\right]
$$

The method that was described in this section thus allows the prediction of the convergence error through Eq. (47) (reproduced below) for a sampled velocity component:

$$
\tilde{\sigma}^{2}(V) \approx \frac{\hat{\mu}_{1}^{k, 2}(\Sigma V)}{\hat{\mu}_{k, 1}^{4}(N)}{\widehat{\tilde{\sigma}^{2}}}^{k}(N)+\frac{{\widehat{\tilde{\sigma}^{2}}}^{k}(\Sigma V)}{\hat{\mu}_{k, 1}^{2}(N)}-\frac{2 \hat{\mu}_{1}^{k}(\Sigma V)}{\hat{\mu}_{1}^{k, 3}(N)} \widehat{\widetilde{\sigma}}^{k}(\Sigma V, N) .
$$

by using Eq. (83), to calculate ${\widehat{\tilde{\sigma}^{2}}}^{k}(N)$ as follows:

$$
{\widehat{\tilde{\sigma}^{2}}}^{k}(N) \approx \hat{\mu}_{1}^{k}(N)\left[\frac{2}{\mathcal{C}}-1\right]
$$

by using Eq. (101), to calculate ${\widehat{\widetilde{\sigma}^{2}}}^{k}(\Sigma V)$ according to:

$$
{\widehat{\tilde{\sigma}^{2}}}^{k}(\Sigma V) \approx \hat{\mu}_{1}^{k}\left(\Sigma V_{x}^{2}\right)\left[\frac{2}{\mathcal{C}_{\Sigma V_{x}}}-1\right]
$$


and by using Eq. (106), to calculate $\widehat{\tilde{\sigma}}^{k}(\Sigma V, N)$ following:

$$
\widehat{\tilde{\sigma}}^{k}(\Sigma V, N) \approx \hat{\mu}_{1}^{k}\left(\Sigma V_{x}\right)\left[\frac{2}{\mathcal{C}}-1\right] .
$$

The quantities that are required for the estimate through Eq. (107) of the convergence error are thus $\hat{\mu}_{1}^{k}(N)$, $\hat{\mu}_{1}^{k}\left(\Sigma V_{x}\right), \hat{\mu}_{1}^{k}\left(\Sigma V_{x}^{2}\right), \hat{\mu}_{1}^{k}(\vec{v})$ and $\hat{\mu}_{1}^{k}(T)$ which are calculated and stored in most DSMC implementations during the sampling period.

\section{Supersonic axisymmetric jet}

\subsection{Simulation setup}

The domain used in the simulation is shown in Fig. 2. A jet with an inlet of radius $0.001 \mathrm{~m}$ is located at $x=0$. The inlet injects argon with number density $8.85 \times 10^{22} \mathrm{~m}^{-3}$ in equilibrium at a temperature of $750 \mathrm{~K}$ with a bulk velocity in the $x$ direction of $510 \mathrm{~m} \mathrm{~s}^{-1}$. This corresponds to a Mach number of $\mathrm{M}=1$ and a Knudsen number of $\mathrm{Kn}=0.01$ (based on the inlet radius). A supersonic outflow boundary condition is used for the top and right sides of the domain. As the flow normal to the top boundary is not supersonic, a modeling error is introduced. Due to the flow temperature, the error introduced is however expected to be small for most of the boundary. A de facto symmetry condition along the centerline is enforced by the axisymmetric move procedure [15].

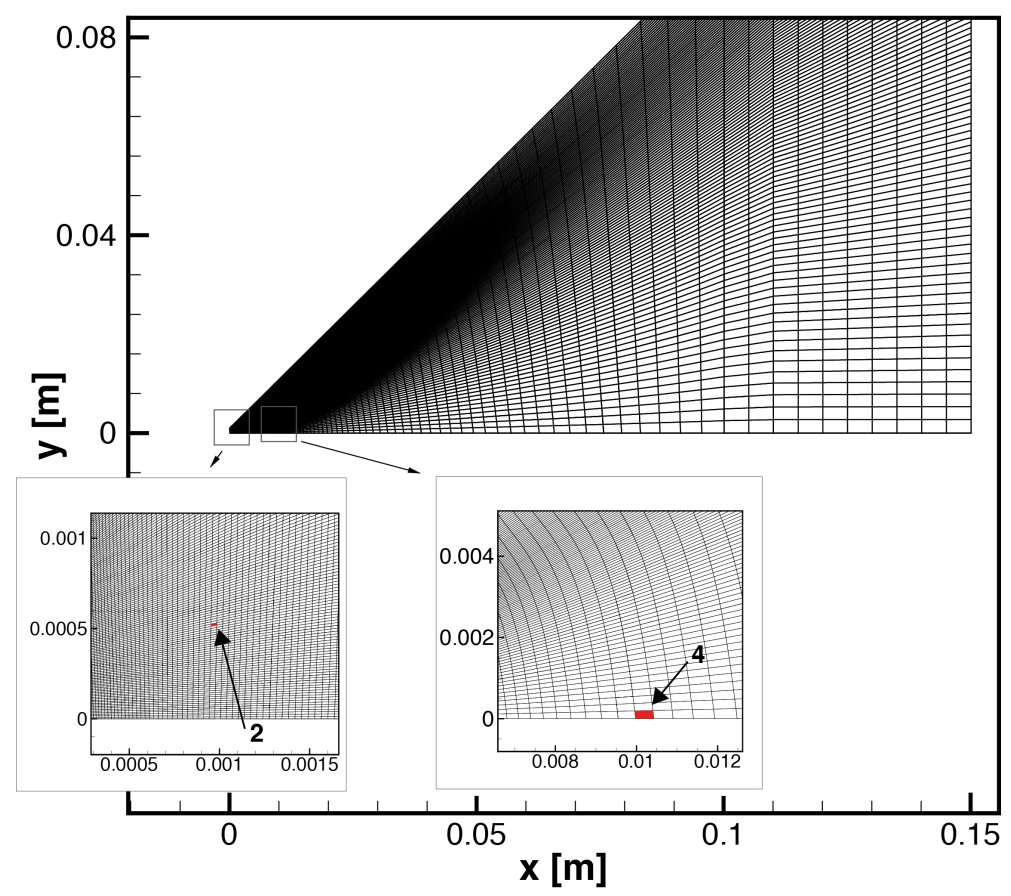

Figure 2: Location and numbering of cells studied in detail.

Details concerning the implementation of the DSMC procedure can be found in section 2.1. The different test cases that are run during the course of this study are summarized in Table 1 which indicates the corresponding $\Delta t$ and $W_{p}$ values that are used. The time step satisfies DSMC requirements for all cases [25]. A view of the average number of particles obtained for all cells for test case C64 is shown in Fig. 3. The test cases will be referred to by the name indicated in the tables in the following. Samples are always taken at every time step once the simulation has reached steady state. In order to gain greater insight into 


\begin{tabular}{|l|l|c|c|}
\hline Test Case & Number of particles $(\mathbf{M})$ & $\mathbf{W}_{\mathbf{p}}$ & $\Delta t[\mathrm{~s}]$ \\
\hline C128 & 67.0 & $2.8 \times 10^{8}$ & $5.0 \times 10^{-9}$ \\
C64 & 33.5 & $5.6 \times 10^{8}$ & $5.0 \times 10^{-9}$ \\
C64050 & 33.5 & $5.6 \times 10^{8}$ & $2.5 \times 10^{-9}$ \\
C64200 & 33.5 & $5.6 \times 10^{8}$ & $1.0 \times 10^{-8}$ \\
C32 & 16.7 & $11.2 \times 10^{8}$ & $5.0 \times 10^{-9}$ \\
C16 & 8.4 & $22.4 \times 10^{8}$ & $5.0 \times 10^{-9}$ \\
\hline
\end{tabular}

Table 1: Test cases to investigate the effect of the particle count on the numerical error.

the simulation, instantaneous DSMC fields are examined in detail by outputting them at every time step for a few selected cells of the domain shown in Fig. 2.

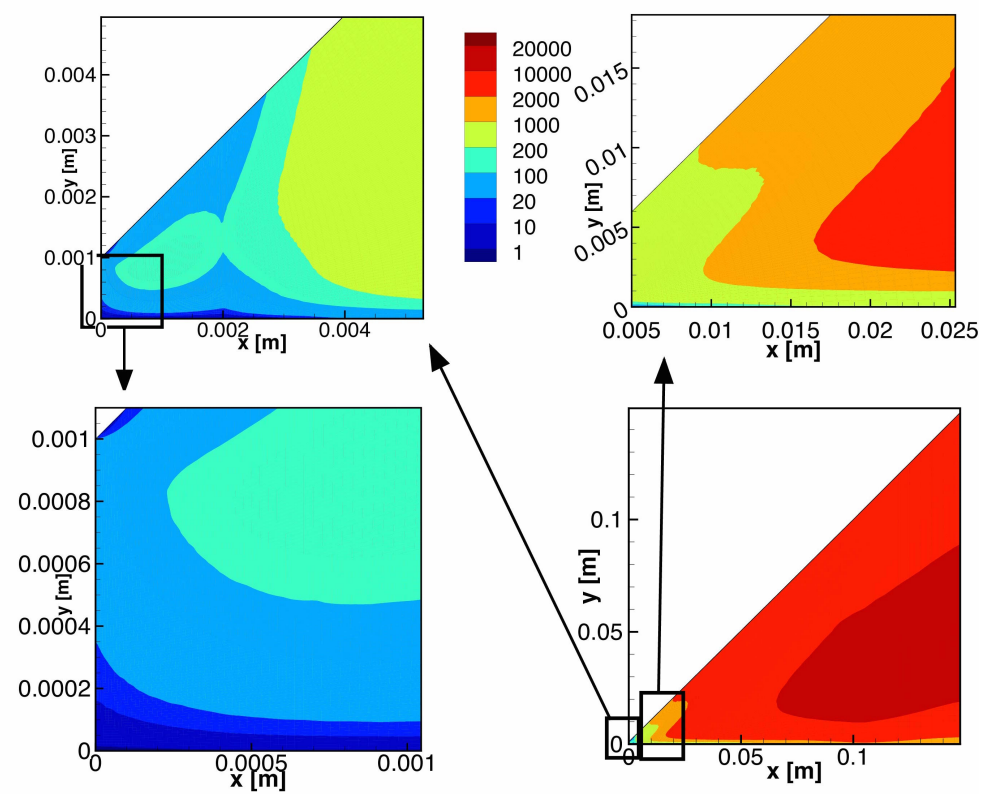

Figure 3: Average number of particles in each cell for test case C64. The cell with the smallest average number of particles is located at the corner of the centerline and the inlet and contains 4.19 .

\subsection{Time correlation between samples}

The time correlation between sampled $N^{k}, \Sigma V^{k}$ and $\bar{V}^{k} \triangleq \frac{\Sigma V^{k}}{N^{k}}$ can be quantified by evaluating their autocorrelation function as outlined in section 3.2.1. Figures $4(\mathrm{a})$ to $4(\mathrm{~b})$ show $\rho_{N}(k), \rho_{\Sigma V_{x}}(k), \rho_{\bar{V}_{x}}(k)$ and $\rho_{\Sigma V, N}(k)$ for two cells. The predicted correlation spectrum for $N$ is obtained with Eq. (83), while that for $\Sigma V_{x}$ is calculated with Eq. (107). As will be later seen, the value of the first correlation coefficient at $k=1$ is well captured by the approximate method. The overall shape of the correlation spectrum is generally well captured for zones with low time correlation (such as cell 2 here). For zones with high levels of correlation, such as cell 4 , the time correlation is always overestimated. We note in passing that the shape of the correlation function is not universal in the domain. Cell 2, for instance, exhibits almost a power law spectrum $\left(\rho \sim k^{-\alpha}\right)$ while that of cell 4 is linear for most wave numbers. This partially explains why our simplified model (which always produces $\rho \sim(1-C)^{k}$ ) is not able to capture all spectra well. Large spatial variations in the extent of the time correlation of $N$ are observed throughout the domain. This is visible 


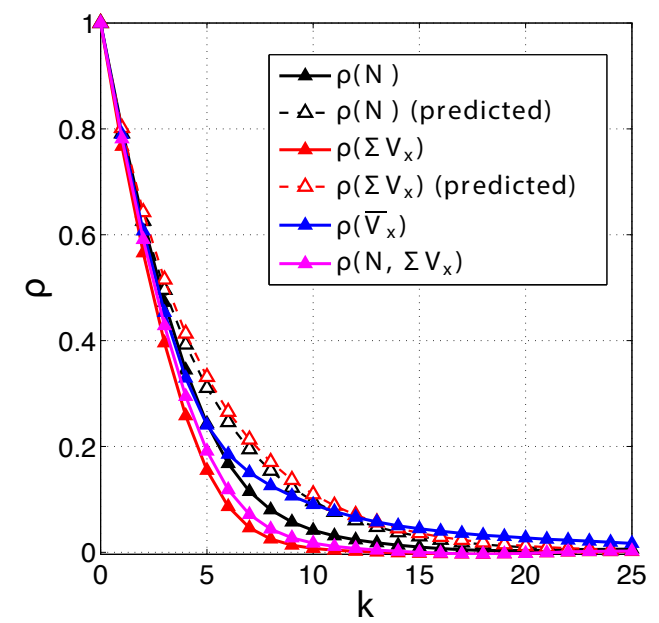

(a) Cell 2

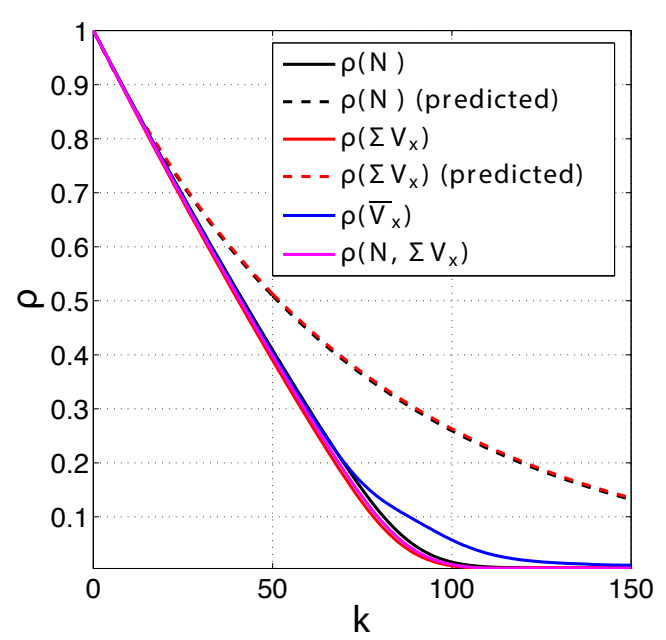

(b) Cell 4

Figure 4: Autocorrelation and correlation functions for the instantaneous velocity and number density as a function of the time step. The predicted spectra $\rho(N)$ are obtained with Eq. (73) and $\rho\left(\Sigma V_{x}\right)$ with Eq. (99) while all other spectra are those observed during the simulation. $\rho\left(N, \Sigma V_{x}\right)(k)$ is symmetric with respect to $k$ for both cells.

in Fig. 5 which displays the observed $\rho_{n}(k=1)$ value. The accuracy of the prediction for the first time correlation coefficient is shown in Figs. 6(a) and 6(b) which display the ratio of the predicted to the actual value of $\rho_{n}(k=1)$ and $\rho_{V_{x}}(k=1)$, respectively. General good agreement (to within $15 \%$ for number density and $20 \%$ for velocity) is obtained for most of the domain. The relatively poor results obtained close to the top part of the inlet are explainable by the small correlation observed in that region for which the model does not perform well due to the linear flux and full correlation assumptions. Poor results are also obtained right next to the inlet which does not satisfy the spatial homogeneity assumption. The large advantage of having derived an analytical model for the first time correlation coefficient $\rho_{1}$ (even if it is not fully accurate) is that it helps to explain the correlation trends that are observed in the domain in Fig. 5. When $u_{x} \gg u_{y}$ and $s_{x} \gg 1$, such as in the downstream portion of the jet because of the expansion, Eq. (70) can well be approximated by $\rho_{N}=1-\frac{u_{x} \Delta x}{\Delta t}$ as $u_{x, i, j}^{+}\left(s_{x}\right) \rightarrow u_{x} \gg \underline{u}_{y}$ and $u_{x, i, j}^{-}\left(s_{x}\right) \rightarrow 0$. The large correlation increase in the downstream portion of the jet is thus explainable by the very large increase in the $\Delta x$ in the downstream direction which far exceeds that of the $x$ bulk velocity $u_{x}$. The drop in correlation observed at $x=0.002$ is explainable by the non-uniformity of the mesh at that location that is visible in the close up in Fig. 5. At that location, the longest side of the cells go from being roughly aligned with bulk flow velocity to being perpendicular to it. This creates a mixing-like phenomenon of particles which results in a lower correlation. We note that the approximate correlation method does not capture the effect of the mesh discontinuity well, as seen in Figs. 6(a) and 6(b), because it is derived for a Cartesian mesh with uniform spacing.

\subsection{Convergence study for $\hat{\mu}_{1}^{k}(n)$}

To obtain results for the convergence of the sampled mean for number density, the convergence error defined by Eq. (17) is calculated by running $N_{\text {sim }}=22$ distinct simulations for the C64 test case. The standard deviation of the ensemble average is then normalized by the standard deviation of the number density $\sigma(n)=\frac{W_{p}}{V} \sigma(N)$ (c.f. Eq. (11)) in the same cell and plotted in black against the number of time steps in a log-log plot. The plots are shown in Fig. 7 for two representative cells shown in Fig. 2. For each cell, the autocorrelation functions for $N$ that was previously shown are used to calculate the predicted normalized standard deviation with Eq. (32) using $K=20$ for Cell 2 and $K=100$ for Cell 4 . The prediction 

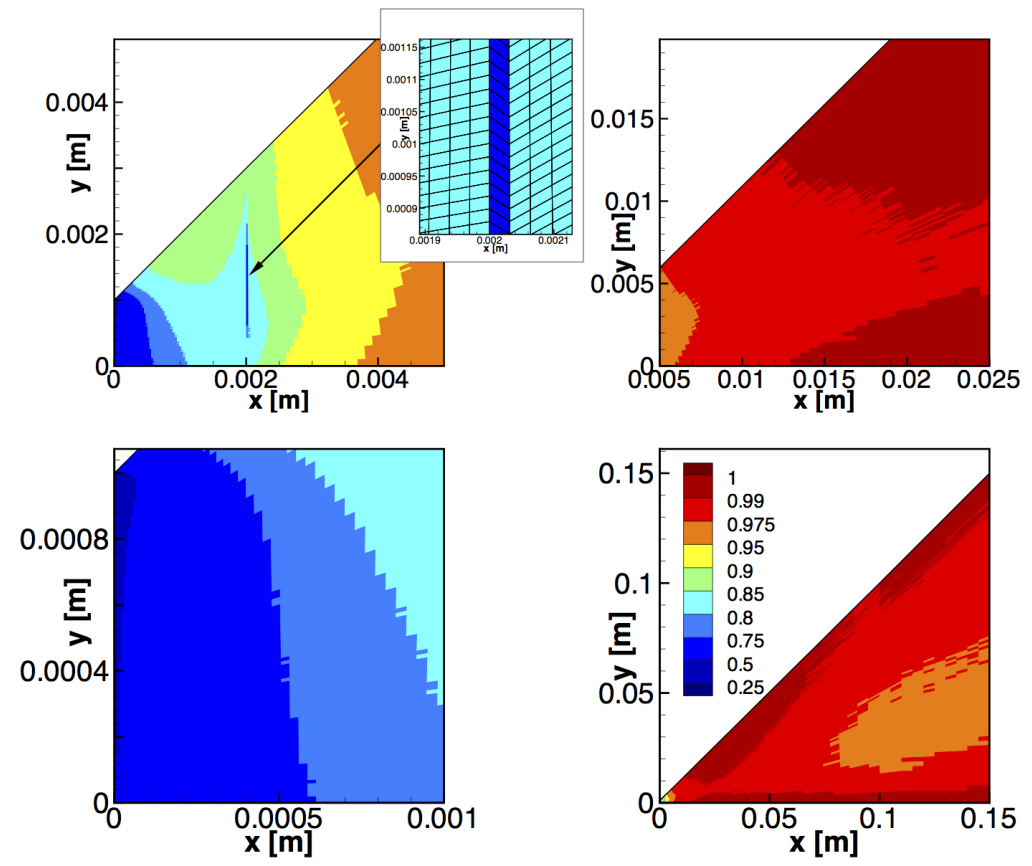

Figure 5: Spatial view of observed $\rho_{N}(k=1)$ for test case C64.

is plotted in red in Fig. 7. If the Central Limit Theorem were to hold perfectly, the ensemble average of the standard deviation would be $\sigma(n) \times k^{-0.5}$. This is because the Central Limit Theorem states that $\hat{\mu}_{1}^{k}(n) \sim \mathcal{N}\left(\mu_{1}(n), \sigma^{2}(n) / k\right)$ if samples are iid. In this case, the ensemble average would of course have a standard deviation of $\frac{\sigma(n)}{k^{1 / 2}}$ so that the normalized standard deviation of the ensemble average would be $\tilde{\varepsilon}_{0}=k^{-1 / 2}$. The increase in standard deviation predicted by Eq. (32) describes very well that observed in practice for all cells, as shown in Fig. 7, as one would expect given the fundamental nature of the Central Limit Theorem. The predicted value of the convergence error following Eq. (83) gives a satisfactory approximation of the convergence error observed in practice. The value of the error is overestimated by $12 \%$ for cell 2 and $30 \%$ for cell 4 .

\subsection{Convergence study for $\hat{\mu}_{1}^{k}\left(V_{x}\right)$}

The same procedure is applied to the study of the convergence of the sampled mean of $V_{x}$ and results are shown in Fig. 8. The predicted theoretical normalized variance of Eq. (46) is plotted in red in the same figure for each cell while that obtained with the approximate method by using Eq. (83) is shown in blue. Very good agreement between the theoretical and observed convergence error is seen for all cells which validates the approximation of the delta method. As for the value of the number density in the previous section, the approximate method is observed to provide an acceptable estimate of the convergence error by overestimating it by $16 \%$ for cell 2 and by $32 \%$ for cell 4 .

\subsection{Implementation considerations of the a priori determination of convergence error}

Within the framework of our test case, the previous section has clearly highlighted that an almost perfect prediction of the convergence error for sampled number density and velocity can be obtained through Eqs. (32) and (46), respectively, by calculating and sampling the correlation spectrum in all cells of the domain. The computational and storage requirements of the implementation of an a priori determination of the value of the convergence error are small compared to those of the underlying simulation. As previously mentioned, it, however, is not straightforward to implement in most existing DSMC codes and necessitates 

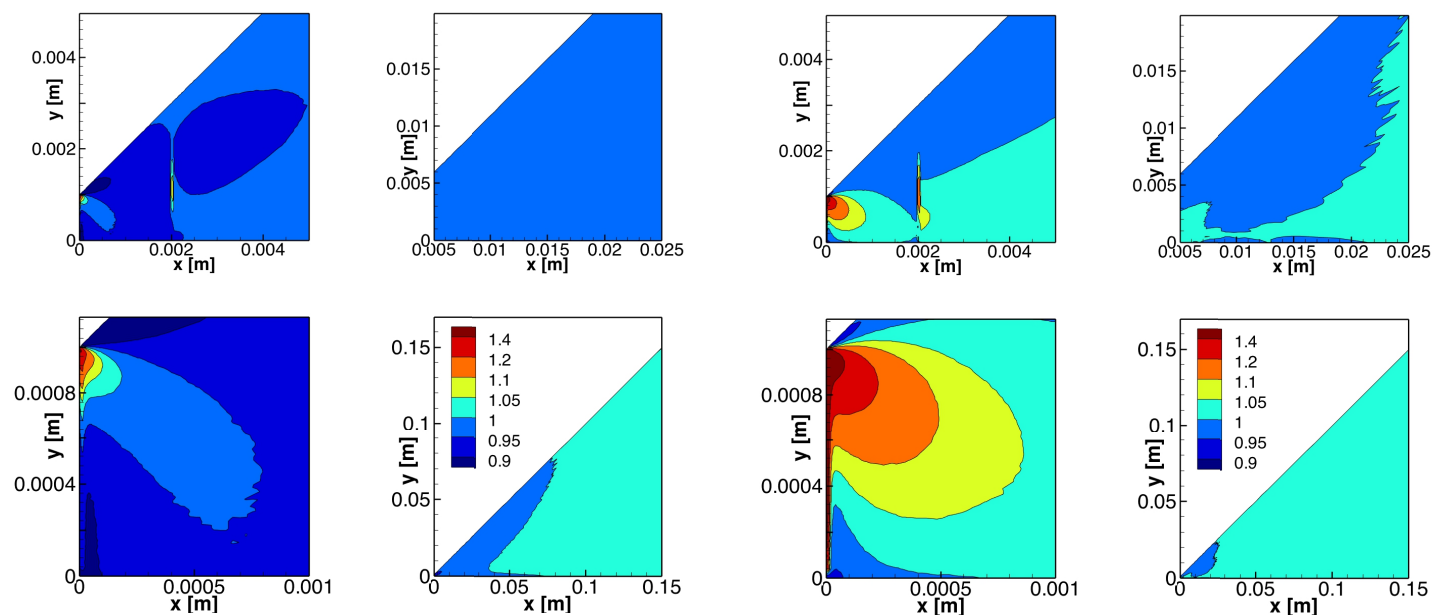

(a) Number of particles: $\rho_{N}$

(b) x-velocity sum: $\Sigma V_{x}$

Figure 6: Ratio of the predicted to the actual value of the autocorrelation spectrum $\rho(k=1)$

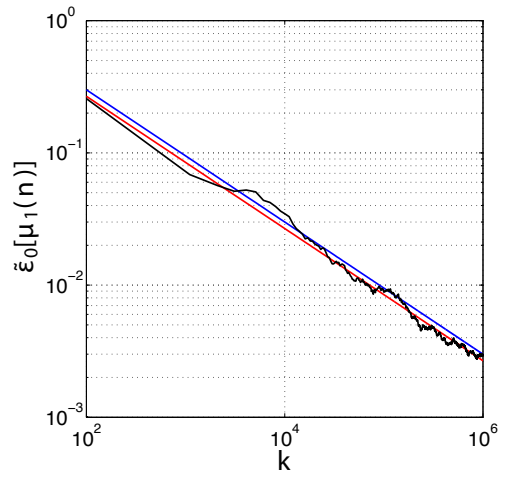

(a) Cell 2

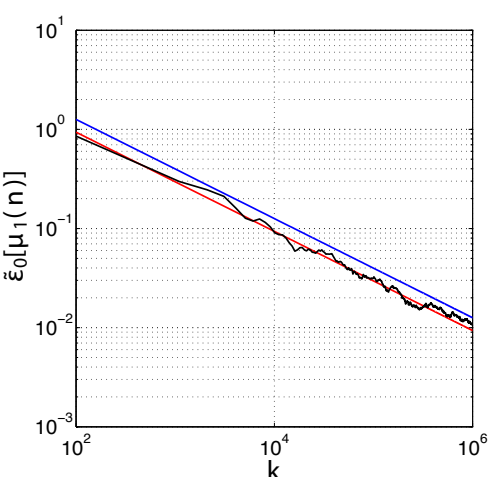

(b) Cell 4

Figure 7: Observed normalized convergence error for sampled number density, $\tilde{\varepsilon}_{0}^{k}\left[\mu_{1}(n)\right]=\frac{\tilde{\varepsilon}^{k}\left[\mu_{1}(n)\right]}{\sigma(n)}$ (black), theoretical prediction of $\frac{\tilde{\sigma}(n)}{k^{1 / 2} \sigma(n)}$ through Eq. (32) (red) and approximate predicted value of Eq. (83) (blue) as a function of the number of sampling steps $k$. 


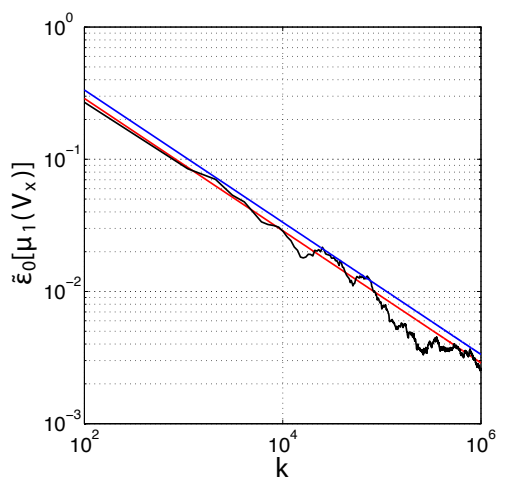

(a) Cell 2

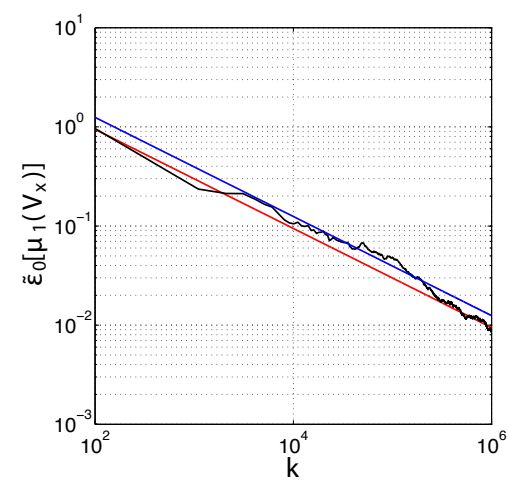

(b) Cell 4

Figure 8: Observed convergence error for sampled $V_{x}, \tilde{\varepsilon}_{0}^{k}\left[\mu_{1}\left(V_{x}\right)\right]=\frac{\tilde{\varepsilon}^{k}\left[\mu_{1}\left(V_{x}\right)\right]}{\tilde{\sigma}_{0}\left(V_{x}\right)}$ (black), theoretical prediction of $\frac{\tilde{\sigma}\left(V_{x}\right)}{k^{1 / 2} \tilde{\sigma}_{0}\left(V_{x}\right)}$ through Eq. (46) (red) and approximate predicted value of Eq. (83) (blue) as a function of the number of sampling steps $k$. $\tilde{\sigma}_{0}\left(V_{x}\right)$, the theoretical value of $\tilde{\sigma}\left(V_{x}\right)$ without correlation, is obtained with Eq. (51).

extra memory that could be used to store additional particles and thus increase accuracy. The approximate correlation method introduced in Section 4 was observed to give a reasonable upper bound for the convergence error. Its relative simplicity combined with the advantage of not having to calculate or store correlation spectra make it a a good method to implement in existing DSMC codes to reduce the uncertainty associated with the lack of convergence of sampled quantities in simulations. Because of the definition of the convergence error, $\tilde{\varepsilon}^{k, 2}\left[\mu_{1}(n)\right]$, following Eq. (17), the distribution function of the sampled number density $\hat{\mu}_{1}^{k}(n)$ follows a normal distribution centered around the converged value and with a variance equal to the convergence error squared:

$$
\hat{\mu}_{1}^{k}(n) \sim \mathcal{N}\left(\mu_{1}(n), \tilde{\varepsilon}^{k, 2}\left[\mu_{1}(n)\right]\right)
$$

This can be used to determine confidence intervals for the sampled mean instance to ensure that $\hat{\mu}_{1}^{k}(n)$ is within a prescribed tolerance of the converged value. From Eq. (111), the probability that $\left|\hat{\mu}_{1}^{k}(n)-\mu_{1}(n)\right|$ is less than $\varepsilon_{\text {tol }}$ is [23]:

$$
P\left(\frac{\left|\hat{\mu}_{1}^{k}(n)-\mu_{1}(n)\right|}{\mu_{1}(n)}<\varepsilon_{\text {tol }}\right)=\operatorname{erf}\left(\frac{\varepsilon_{\text {tol }} \mu_{1}(n)}{\sqrt{2} \tilde{\varepsilon}^{k}\left[\mu_{1}(n)\right]}\right)
$$

Following the definition of Eq. (33) and using the normalization of Fig. 7:

$$
\tilde{\varepsilon}^{k}\left[\mu_{1}(n)\right]=\frac{\tilde{\sigma}(n)}{k^{1 / 2}}=\frac{C_{\tilde{\varepsilon}} \sigma(n)}{k^{1 / 2}} .
$$

The value of constant $C_{\tilde{\varepsilon}}$ for cell 2 of test case C64 is reported in Table 2, as obtained by the theoretical prediction, the approximate method and when ignoring sample correlation. The values of $C_{\tilde{\varepsilon}}$ are given by the $y$ intercept of the corresponding line in Fig. 7. Inserting Eq. (113) into Eq. (112) while requiring that the prescribed tolerance be reached with a specified $P_{\min }$ probability, i.e. $P\left(\left|\hat{\mu}_{1}^{k}(n)-\mu_{1}(n)\right|<\varepsilon_{\text {tol }}\right)>P_{\text {min }}$, one obtains the minimum number of samples required:

$$
k_{\min }=\left[C_{\tilde{\varepsilon}} \frac{\sigma(n)}{\mu_{1}(n)} \frac{\operatorname{erf}^{-1}\left(P_{\min }\right)}{\varepsilon_{\mathrm{tol}}}\right]^{2}
$$

The numerical values obtained for $k_{\min }$ necessary to be $P_{\min }=95 \%$ certain that the convergence error $\frac{\left|\hat{\mu}_{1}^{k}(n)-\mu_{1}(n)\right|}{\mu_{1}(n)}$ is less than $\varepsilon_{\text {tol }}=1 \%$ with the three different approaches are reported in Table 2 . It is interesting to note the large effect of the correlation of samples on the minimum number of steps required. This effect would, of course, be entirely missed if the "naive" Central Limit Theorem, without correlation correction, were applied to determine convergence. 


\begin{tabular}{|l|l|c|c|}
\hline & Theory & Approximate method & No correlation \\
\hline$C_{\varepsilon}$ & 2.68 & 3.00 & 1.0 \\
$k_{\min }$ & 2,602 & 3,260 & 362 \\
\hline
\end{tabular}

Table 2: Comparison of the minimum number of samples obtained with Eq. (114) for cell 2 for test case C64 for which $\frac{\sigma(n)}{\mu_{1}(n)}=0.097$.

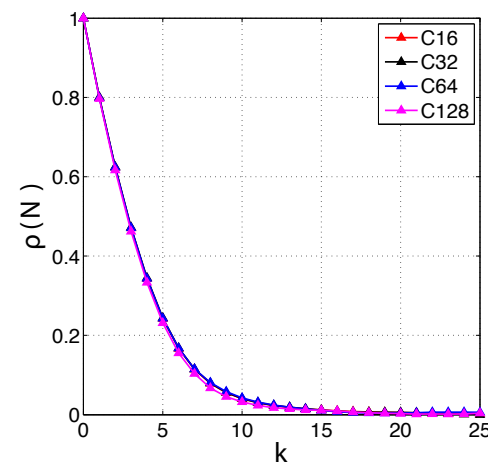

(a) Cell 2

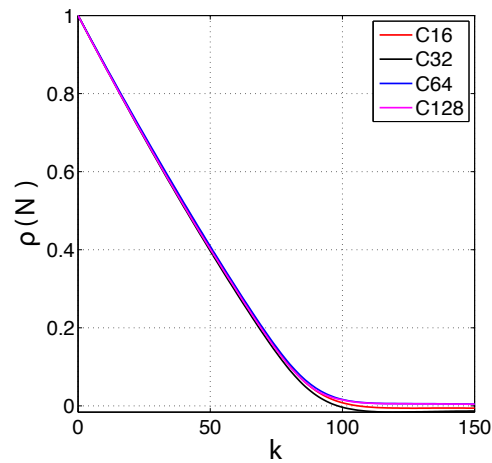

(b) Cell 4

Figure 9: Influence of the particle count on the autocorrelation function for $N$.

\subsection{Effect of numerical parameters on the convergence error}

Having previously determined the general behavior of the convergence error for a baseline simulation, i.e. test case C64, the aim of the present section is to determine the influence of the number of particles and time step.

The impact of the number of particles in the simulation on the autocorrelation function is evaluated by calculating it for test cases C16, C32, C64 and C128 for all cells of the simulation. Results obtained for two cells are given in Fig. 9 which shows that the autocorrelation function is unaffected by changes in the particle count. Nearly identical results are obtained for all other cells that are examined. This indicates that the time correlation of samples, normalized by their variance, is independent of particle count. That is to say that, although the variance of $N$ scales as $W_{p}$, as shown by Eq. (12), the shape of its time correlation is maintained across particle counts.

To investigate the effect of the number of particles in the simulation on the rate of convergence of the convergence error, 10 simulations are run for each of the C16 and C256 cases in addition to the 20 simulations for C64. For each particle count, an ensemble average of the convergence error is calculated and is plotted in Fig. 10. For all particle counts, the same convergence rate for the error is observed. The discrepancies between the convergence paths are attributable to the relatively small number of simulations used to calculate the ensemble average for the convergence error. The fact that convergence is independent of particle count is a direct consequence of the invariance of the autocorrelation function. The exact same result is obtained with the approximate model of Section 4. As the Courant number, defined in Eq. (71), is independent of the particle count, the predicted correlation spectrum of Eq. (73) is maintained across particle counts.

The effect of varying the time step on the correlation of samples and rate of convergence is now investigated. This is accomplished by running three simulations referred to in Table 1 by C64050, C64 and C64200 corresponding, respectively, to $\frac{\Delta t_{0}}{2}, \Delta t_{0}$ and $2 \Delta t_{0}$. The autocorrelation function for $N$ that is obtained for each test case is shown in Fig 11 as a function of $\tilde{k}=k \frac{\Delta t}{\Delta t_{0}}$. The autocorrelation functions are observed to collapse to the same curve under this change of variable. This indicates that

$$
\rho_{N}(k, \Delta t)=\rho_{N, 0}(\tilde{k})
$$




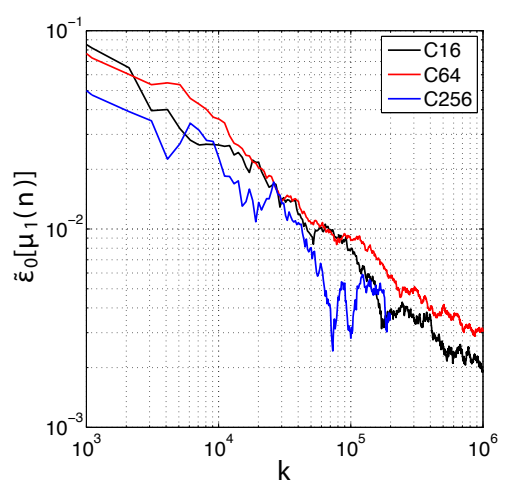

(a) Cell 2

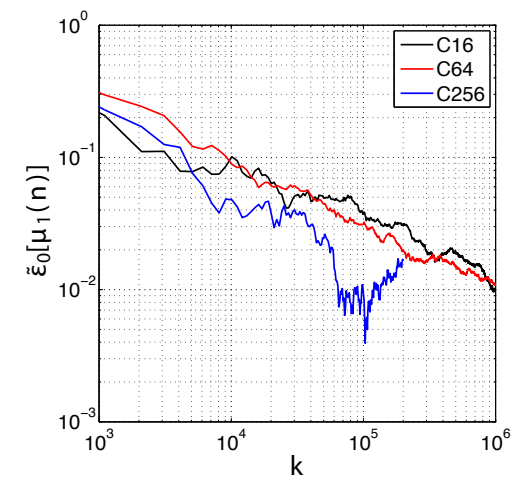

(b) Cell 4

Figure 10: Observed normalized convergence error for sampled $n, \tilde{\varepsilon}_{0}^{k}\left[\mu_{1}(n)\right]=\frac{\tilde{\varepsilon}^{k}\left[\mu_{1}(n)\right]}{\sigma(n)}$, as a function of the number of samples for multiple particle counts.

where $\rho_{N, 0}$ is independent of both $k$ and $\Delta t$. The invariance of the correlation function under this scaling quantifies the reduced or increased time correlation observed between samples when the time step is raised or lowered. This scaling of the spectrum with $\Delta t$ is indeed very well predicted by the approximate model for small correlation values, such as observed in cell 4 in Fig. 11(b). The scaling for higher correlation values, such as cell for cell 2, holds less well, as seen in Fig. 11(a). This is due to the form of the approximate correlation spectrum which, according to Eq. (71), is of the form $\rho(k)=\left(1-\frac{u}{\Delta x} \Delta t\right)^{k}$ (only considering the $\mathrm{x}$ velocity for conciseness) which can be expanded via Taylor series as follows:

$$
\begin{aligned}
& \rho_{N}(k, \Delta t)=\left(1-\frac{\underline{u} \Delta t}{\Delta x}\right)^{k}=\left(1-\frac{\underline{u} \Delta t \Delta t_{0}}{\Delta x \Delta t_{0}}\right)^{k}=1-\frac{k \underline{u} \Delta t \Delta t_{0}}{\Delta x \Delta t_{0}}+\mathcal{O}\left(\frac{\underline{u} \Delta t \Delta t_{0}}{\Delta x \Delta t_{0}}\right)^{2} \\
& =\left(1-\frac{\underline{u} \Delta t_{0}}{\Delta x}\right)^{k \frac{\Delta t}{\Delta t_{0}}}+\mathcal{O}(\mathcal{C})^{2}=\rho_{N, 0}\left(k \frac{\Delta t}{\Delta t_{0}}\right)+\mathcal{O}(\mathcal{C})^{2}
\end{aligned}
$$

with $\rho_{N, 0}\left(k^{\prime}\right)=\left(1-\frac{u \Delta t_{0}}{\Delta x}\right)^{k^{\prime}}$. This means that the spectra produced by the approximate method with different time steps only reduce by change of variable to the same scaling for small Courant numbers which explains the discrepancies observed in Fig. 11(a) for cell 2 where the $\mathcal{C}$ number is 0.21 vs. 0.013 for cell 4 . The effect of the time step on the evolution of the convergence error is investigated by calculating its ensemble average for the three aforementioned test cases. The ensemble average is calculated with 10 simulation for both C64050 and C64200 while 20 simulations are used for C64. The evolution of the ensemble average, as well as the prediction obtained from Eq. (32) by using the spectrum shown in Fig. 11, is shown for selected cells in Fig. 12. The prediction of the approximate model is also plotted for the three different time steps. As previously in the case of $\Delta t_{0}$, the predicted value for the convergence error is larger than that actually for observed for $\Delta t_{0}$ and $2 \Delta t_{0}$ which is due to the overestimation of the correlation spectrum as shown in Fig. (11).

\section{Hypersonic 2-D cylinder flow}

In order to further establish the generality of the results obtained in the previous section, the analysis of the convergence error is carried out within the framework of a hypersonic blunt body flow. 


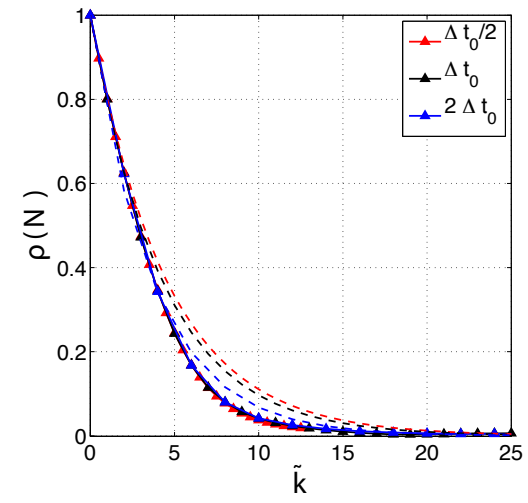

(a) Cell 2

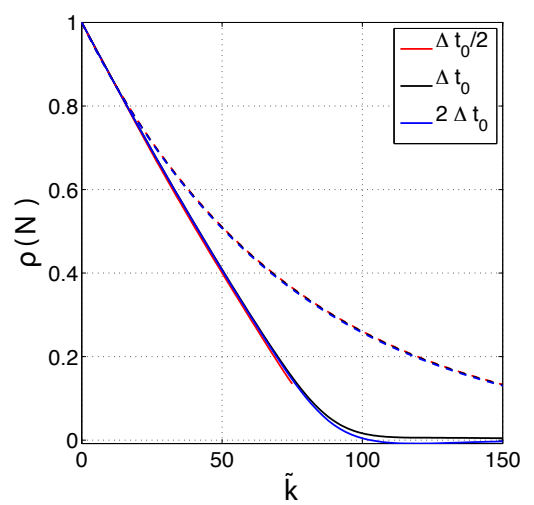

(b) Cell 4

Figure 11: Influence of the time step on the autocorrelation function for $N$, with $\tilde{k}=\frac{k \Delta t}{\Delta t_{0}}$. Solid lines are the observed correlation spectra and dashed lines those predicted by the approximate model.

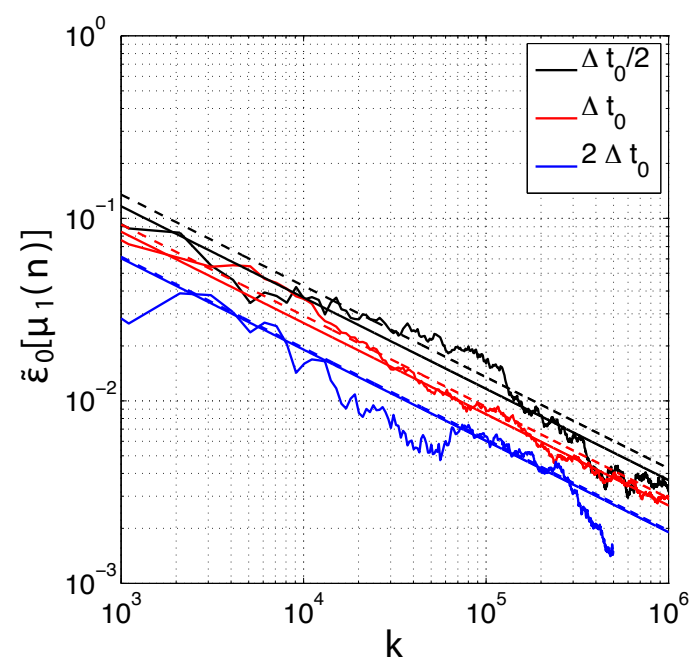

(a) Cell 2

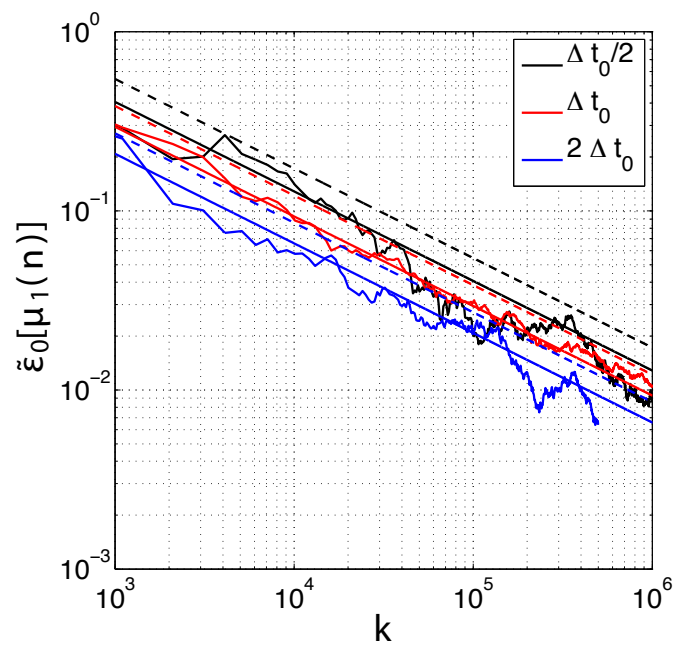

(b) Cell 4

Figure 12: Normalized convergence error for sampled $n, \tilde{\varepsilon}_{0}^{k}\left[\mu_{1}(n)\right]=\frac{\tilde{\varepsilon}^{k}\left[\mu_{1}(n)\right]}{\sigma(n)}$, as a function of the number of samples $k$ for multiple time steps, theoretical prediction of Eq. (32) (solid lines) and approximate model (dashed lines). 


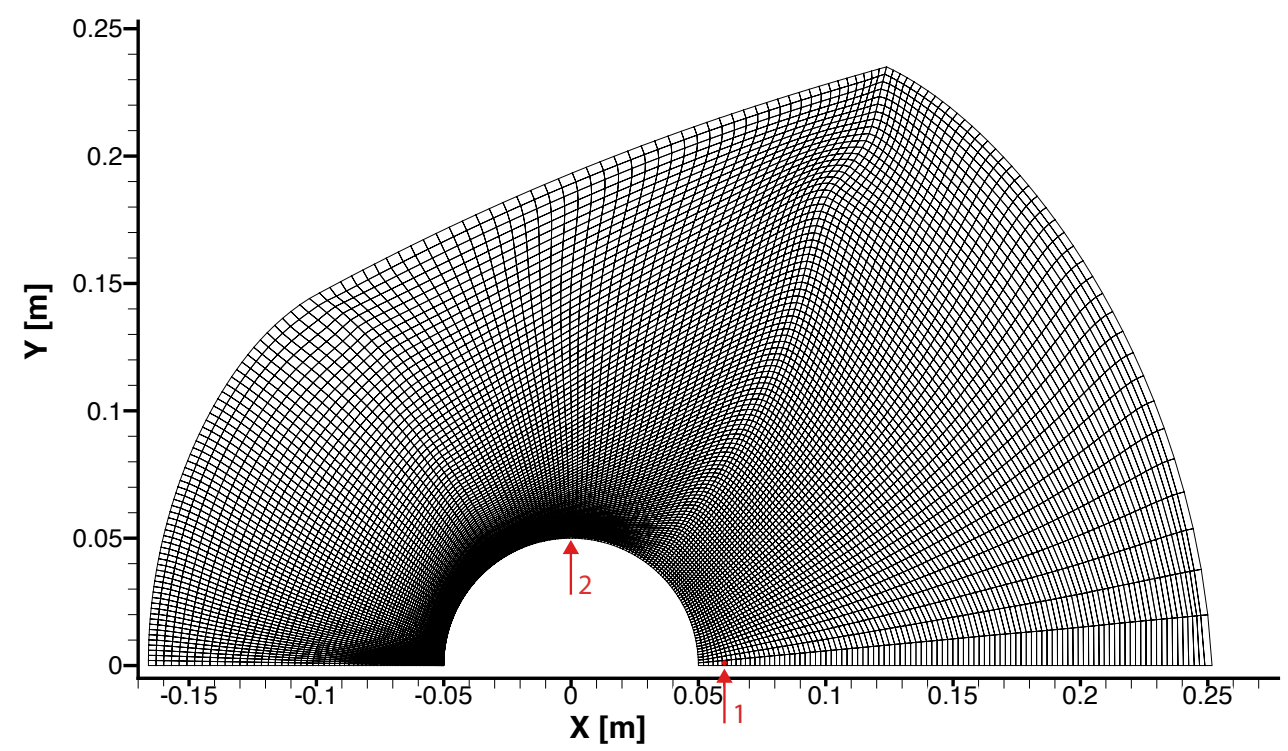

Figure 13: View of the mesh for the hypersonic 2-D cylinder test case. Cell 2 is the first cell off the wall of the cylinder at $x=0$.

\begin{tabular}{|l|l|c|c|}
\hline Test Case & Number of particles (M) & $\mathbf{W}_{\mathbf{p}}$ & $\Delta t[\mathrm{~s}]$ \\
\hline D1 & 3.75 & $6 \times 10^{12}$ & $1.5 \times 10^{-7}$ \\
D2 & 7.5 & $3 \times 10^{12}$ & $1.5 \times 10^{-7}$ \\
D205 & 7.5 & $3 \times 10^{12}$ & $7.5 \times 10^{-6}$ \\
D220 & 7.5 & $3 \times 10^{12}$ & $3.0 \times 10^{-7}$ \\
D4 & 15 & $1.5 \times 10^{12}$ & $1.5 \times 10^{-7}$ \\
\hline
\end{tabular}

Table 3: Numerical parameters of 2-D cylinder test cases.

\subsection{Geometry and flow conditions}

The test case examined in this section consists of a Mach 10 flow around a two-dimensional cylinder with a radius of $5 \mathrm{~cm}$ at a Knudsen number (based on the cylinder diameter) of 0.05 . The simulation domain is depicted in Fig. 13. This flow regime is obtained by using a free stream Argon flow in equilibrium at a temperature of $200 \mathrm{~K}$ with a bulk velocity of $2634 \mathrm{~m} / \mathrm{s}$ and a number density of $2.35 \times 10^{20} \mathrm{~m}^{-3}$. Only the upper part of the flow is simulated as a symmetry boundary condition is used on the centerline. On the cylinder surface, the wall boundary condition consists in full accommodation to a constant temperature of $500 \mathrm{~K}$. Finally, a supersonic outflow boundary condition is used on the right side of the domain. The numerical parameters of the various runs considered in the following are summarized in Table 3. Case D2 corresponds to the baseline case that will be examined in details in Sections 6.2 and 6.3. Others are studied in Section 6.4 to investigate the impact of numerical parameters on the convergence error. As in the previous test case, a constant weight and time step are utilized, while further details about the DSMC implementation can be found in Section 2.1. For test case D2, the time step is chosen so as to satisfy DSMC requirements [25] and the cell with the smallest average number of particles is that located at the forward stagnation point with an average of 21.4. 


\subsection{Time correlation between samples}

Following the approach undertaken with the previous test case, the correlation is examined both globally and in detail for two representative cells of the simulation, i.e. cells 1 and 2 depicted in Fig. 13 . Figure 14 gives an idea of the spatial variation of time correlation in the simulation by showing the first correlation coefficient for $N$. The inlet boundary conditions contribute to reduce the correlation in the first few rows of cells on the left of the domain. Further past the inlet, the correlation is fairly uniform as the flow and mesh are nearly homogenous. The influence of the wall boundary condition then becomes manifest with a large drop in the correlation close to the cylinder wall. The increase in temperature, despite the reduction in cell size, and the decrease in bulk velocity first contribute to reduce the correlation. In cells closer to the cylinder, the decrease in correlation is due to the influx of particles from the boundary. The particles have their velocity altered at the boundary so as to follow a half-Maxwellian at the prescribed wall temperature. The correlation reduction caused by the wall boundary conditions is further compounded by the effect of collisions which become increasingly important close to the cylinder.

Correlation spectra shapes are very similar to those previously obtained so that the findings of the previous section seem to be rather general. Multiple cells were examined in addition to the two presented here with similar conclusions. However, one interesting result highlighted by this test case is that not all cells have a correlation spectrum that is symmetric with respect to $k$. This lack of symmetry was systematically observed for all cells located close to the boundary of the cylinder, as for instance shown in Fig. 15(b) for cell 2. As the distance to the cylinder increases, the symmetry with respect to $k$ is recovered as illustrated by the case of Cell 1 in Fig. 15(a). This means that the value of the spectrum $\hat{\gamma}_{\Sigma V, N}(k)$ should really be calculated and stored for $k=-N, \ldots 0, \ldots N$ in order to use Eqs. (46) and (44) to determine $\tilde{\Sigma}^{2}(V)$ because no advantage can be taken of the symmetry.

The approximate correlation method detailed in Section 4 is used to calculate correlation coefficients $\rho_{N}(k=1)$ and $\rho_{\sigma V_{x}}(k=1)$ following Eqs. (72) and (99) respectively. The ratio of the value obtained with the approximate method to the actual is plotted in Figs. 16(a) and 16(b). Good agreement is obtained far enough from the boundaries. The approximate method does not perform well for cells close to the front of the cylinder because the wall boundary conditions introduce anisotropy in the flow which is not accounted for in its derivation. This can also be seen in the approximate spectra obtained in Fig. 15(b). However, cells in that region are characterized by particularly low levels of correlation compared to the rest of the flow so that its accurate quantification may be less important. Quantities related to cells close to or on the boundary may, however, be of great importance for many engineering applications in which case the method outlined in Section 3.3 based on the calculation and sampling of a few correlation coefficients would have to be used.

\subsection{Convergence study for $\hat{\mu}_{1}^{k}(n)$ and $\hat{\mu}_{1}^{k}\left(V_{x}\right)$}

The evolution of the convergence error for test case D2 is obtained by calculating the variance of sampled quantities following Eq. (17) amongst $N_{\text {sim }}=20$ different simulations. The results for the sampled number density and velocity of cells 1 and 2 are shown in Figs. 17 and 18 along with its prediction using the full correlation spectra and the approximate correlation method. As expected and previously noted, the use of the full spectrum provides an excellent a priori prediction of the value of the convergence error. The approximate correlation method is seen to provide a good approximation of the convergence error for the two cells in line with what was previously observed.

\subsection{Effect of numerical parameters}

We conclude our study of this test case by investigating the effect of the number of particles and time step on the correlation of samples. This is done by examining the correlation spectrum for individual cells of the simulation, in addition to the baseline case D2, for the other test cases outlined in Table 3 . The correlation spectra are seen in Fig. 19, as was previously noted, to be independent of the number of particles used in the simulation. When the time step is changed, the spectrum scales self-similarly with $\frac{\Delta t_{0}}{\Delta t}$ as shown in Fig. 20. Identical results are obtained for all other cells that are examined which further confirms these results. 


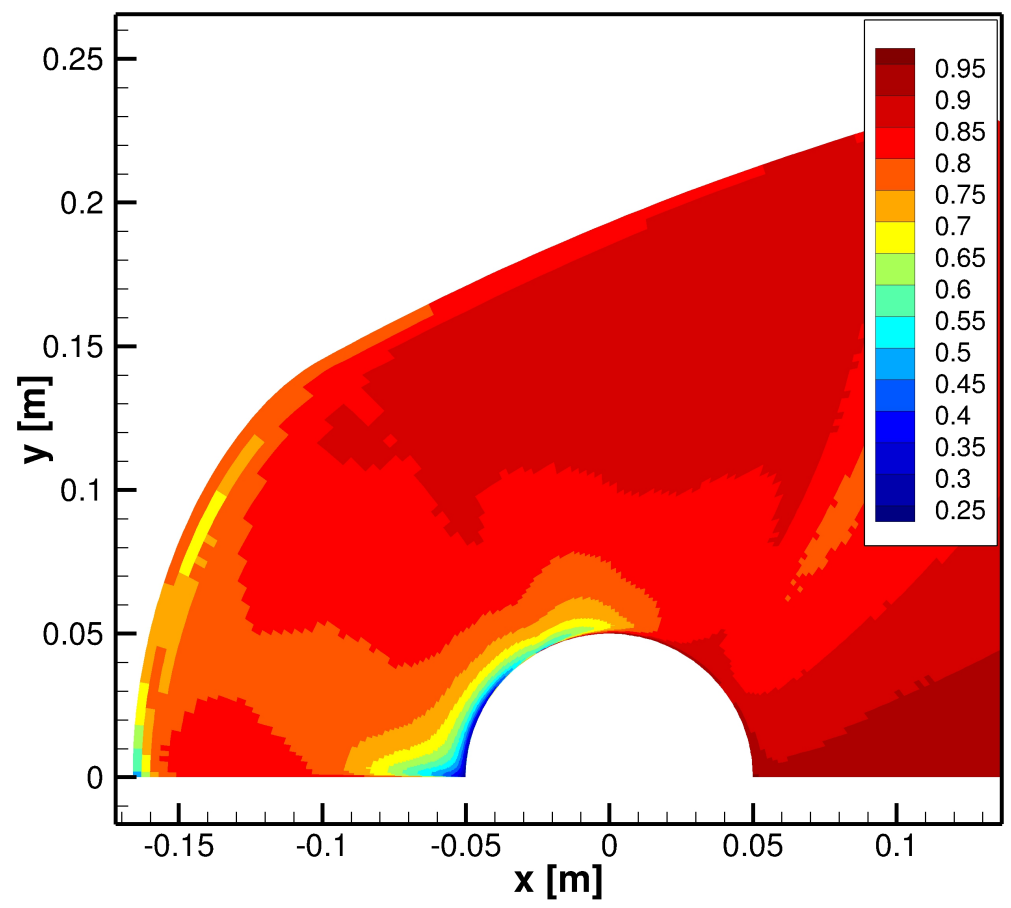

Figure 14: Spatial view of observed $\rho_{N}(k=1)$ for test case D2.

\subsection{Comments on the applicability of the approximate method}

From the results obtained with the approximate correlation method for these two test cases, a few conclusions can be drawn. The method is seen to perform best in zones with high levels of correlation where the convergence error tends to be large. Wherever large flow anisotropies exist, such as close to inflow or wall boundaries, the method, as evidenced by cell 2 of the second test case, performs less well. These zones are often marked by low levels of correlation and thus a lower convergence error so their precise calculation might be less critical. The approximate method, however, typically allows a half order of magnitude improvement in the estimation of the convergence error compared to when sample correlation is neglected (which corresponds to $\tilde{\varepsilon}_{0}^{k}=\frac{1}{\sqrt{k}}$ in all plots of the normalized convergence error) while being trivial to implement. It thereby represents a worthy addition to an existing DSMC code if $a$ quantification of the convergence error is desired. However, in the case when an exact quantification is needed, the appropriate correlation spectra should be calculated and sampled (in whole or in part).

\section{Conclusions}

A framework for the analysis of the convergence of a DSMC simulation was presented and the convergence error was rigorously defined. The convergence error was studied by calculating the ensemble standard deviation of sampled means during their sampling period. It was found to vary as $\tilde{\sigma} k^{-1 / 2}$ for the sampled mean of the number density and velocity. Time correlation between samples is such that the standard Central Limit Theorem cannot be applied for sampled means. The autocorrelation function of sampled quantities was used to quantify the time correlation between samples. Important time correlation was observed in zones characterized by a large Courant-like characteristic number that was introduced. A modified version of the Central Limit Theorem that takes correlation into account for both particle and cell-based variables 


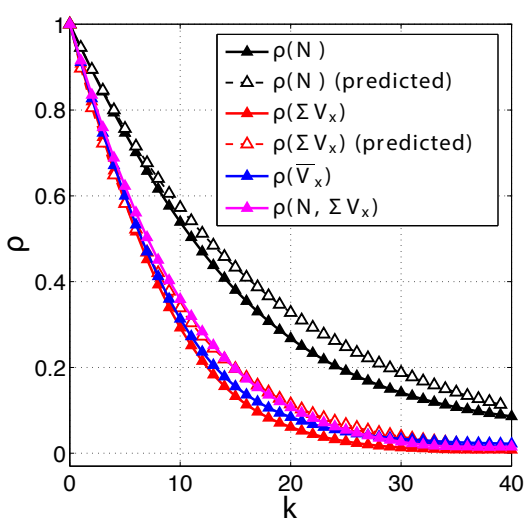

(a) Cell 1

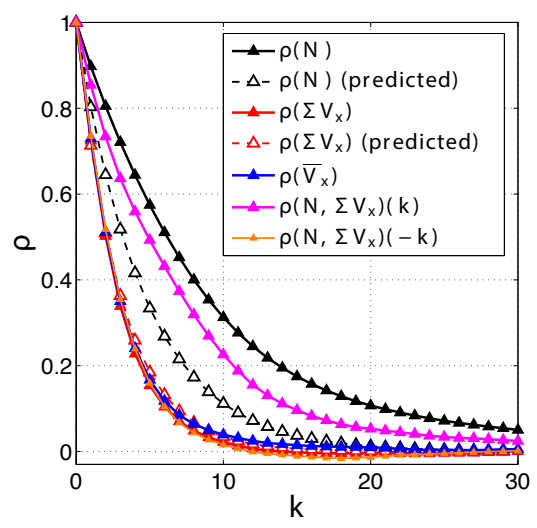

(b) Cell 2

Figure 15: Autocorrelation and correlation functions for the instantaneous velocity and number density as a function of the time step. The predicted spectra $\rho(N)$ are obtained with Eq. (73) and $\rho\left(\Sigma V_{x}\right)$ with Eq. (99) while all other spectra are those observed during the simulation. $\rho\left(N, \Sigma V_{x}\right)(k)$ is symmetric with respect to $k$ for cell 1 .

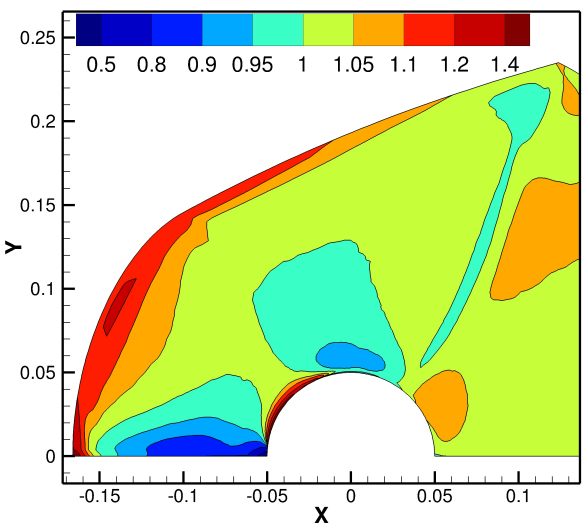

(a) Number of particles: $\rho_{N}$

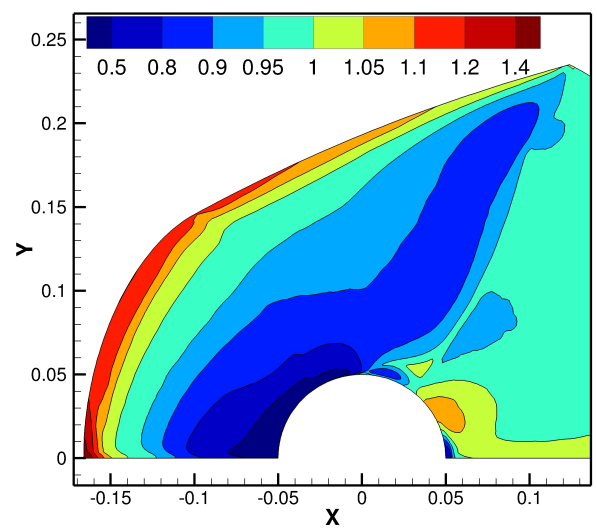

(b) x-velocity sum: $\Sigma V_{x}$

Figure 16: Ratio of the predicted to the actual value of the autocorrelation spectrum $\rho(k=1)$ 


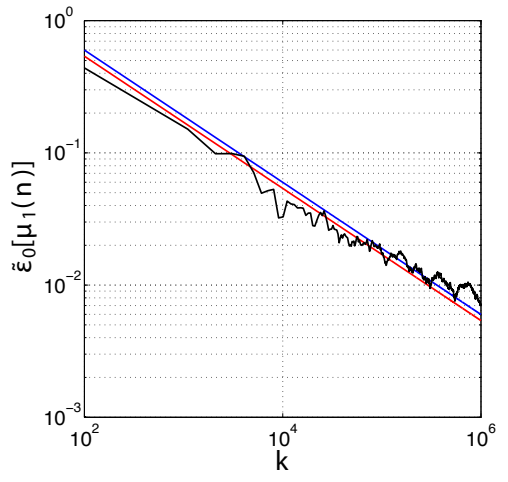

(a) Cell 1

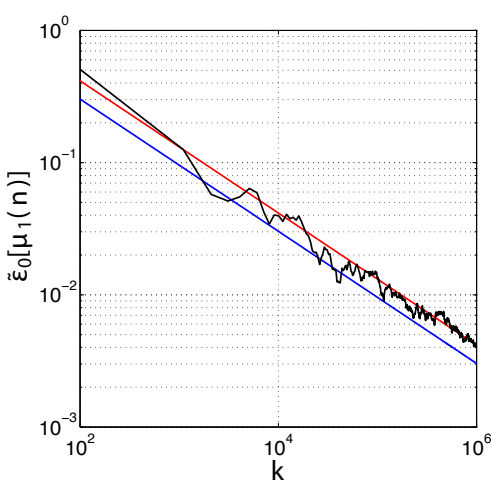

(b) Cell 2

Figure 17: Observed normalized convergence error for sampled number density, $\tilde{\varepsilon}_{0}^{k}\left[\mu_{1}(n)\right]=\frac{\tilde{\varepsilon}^{k}\left[\mu_{1}(n)\right]}{\sigma(n)}$ (black), theoretical prediction of $\frac{\tilde{\sigma}(n)}{k^{1 / 2} \sigma(n)}$ through Eq. (32) (red) and approximate predicted value of Eq. (83) (blue) as a function of the number of sampling steps $k$.

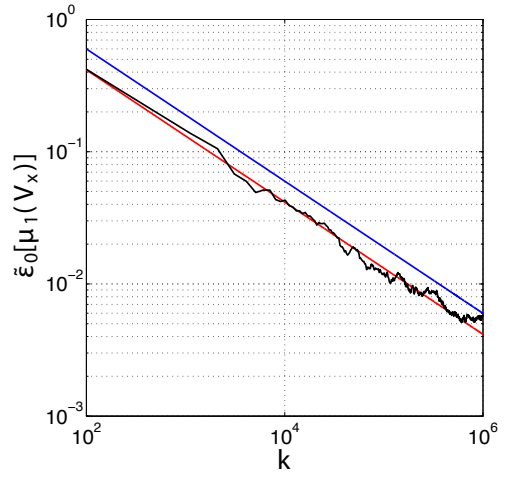

(a) Cell 1

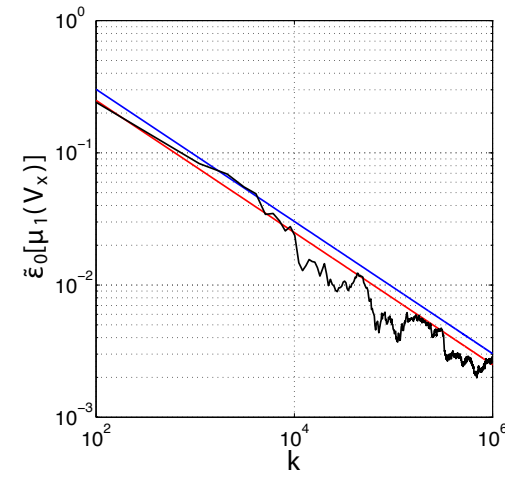

(b) Cell 2

Figure 18: Observed convergence error for sampled $V_{x}, \tilde{\varepsilon}_{0}^{k}\left[\mu_{1}\left(V_{x}\right)\right]=\frac{\tilde{\varepsilon}^{k}\left[\mu_{1}\left(V_{x}\right)\right]}{\tilde{\sigma}_{0}\left(V_{x}\right)}$ (black), theoretical prediction of $\frac{\tilde{\sigma}\left(V_{x}\right)}{k^{1 / 2} \tilde{\sigma}_{0}\left(V_{x}\right)}$ through Eq. (46) (red) and approximate predicted value of Eq. (83) (blue) as a function of the number of sampling steps $k$. $\tilde{\sigma}_{0}\left(V_{x}\right)$, the theoretical value of $\tilde{\sigma}\left(V_{x}\right)$ without correlation, is obtained with Eq. (51). 


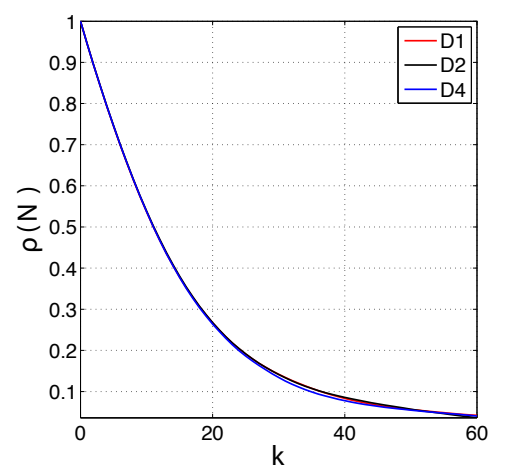

(a) Cell 1

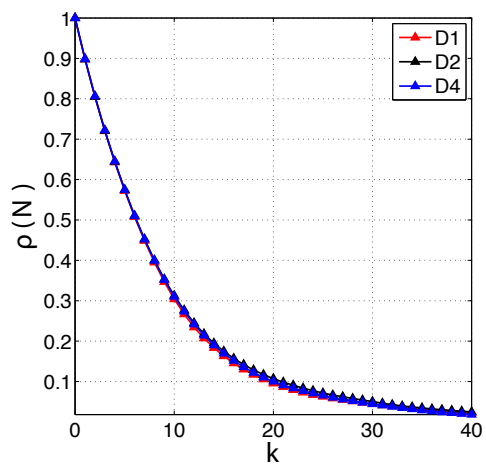

(b) Cell 2

Figure 19: Influence of the particle count on the autocorrelation function for $N$.

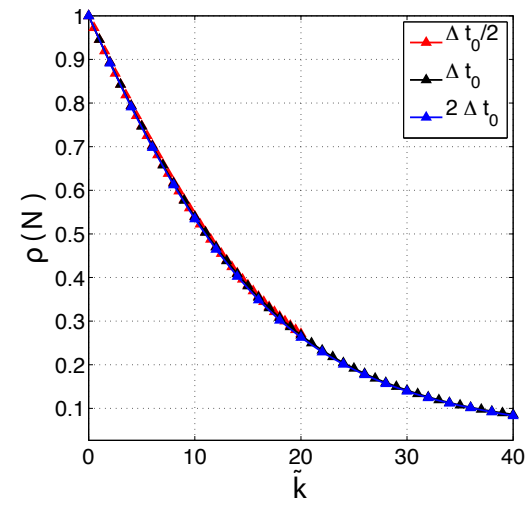

(a) Cell 1

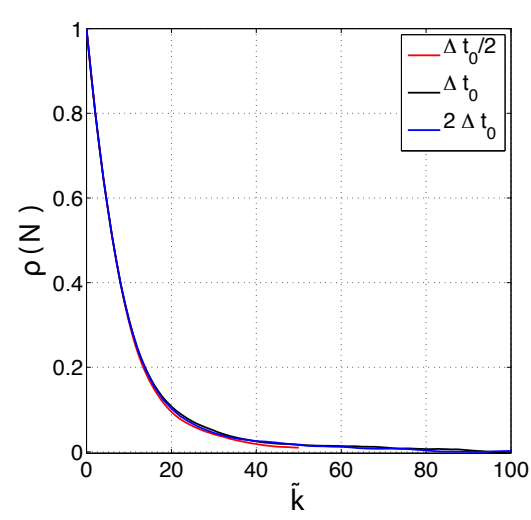

(b) Cell 2

Figure 20: Influence of the time step on the autocorrelation function for $N$, with $\tilde{k}=\frac{k \Delta t}{\Delta t_{0}}$. 


\begin{tabular}{|l|l|c|c|c|c|}
\hline Condition & $u_{x}[\mathrm{~m} / \mathrm{s}]$ & Temperature $[\mathrm{K}]$ & $\Delta t[\mathrm{~s}], \Delta x[m]$ & $\frac{u_{x} \Delta t}{\Delta x}$ & $\mathbb{E}[N]$ \\
\hline A (point 2) & 740 & 450 & $510^{-9}, 3.310^{-5}$ & 0.11 & 106 \\
B (point 4) & 995 & 80 & $510^{-9}, 4.710^{-4}$ & 0.01 & 96 \\
\hline
\end{tabular}

Table 4: Condition for the one dimensional test case of Fig. 21.

(e.g., respectively, $n$ and $V$ ) was presented. It was then used to formulate a framework to a priori predict the value of the convergence error during the course of the simulation. It was found to accurately predict the observed standard deviation $\tilde{\sigma}$ for all cells of the domain that were examined within the framework of an axisymmetric supersonic jet and a two-dimensional cylinder flow. Because of the relative complexity of implementing such a model in a DSMC code, principally the calculation and storage of correlation spectra, an approximate correlation calculation method was developed. Based on a series of simplifying assumptions for fluxes of particles entering and leaving each individual cell, it produces acceptable predictions for the first time correlation coefficient for the two cases examined. The latter is in turn used to approximate the entire spectra which allows, at least within the framework of high speed rarefied flows, an acceptable estimate of the convergence error. The analytical expression produced by the approximate model furthermore allows an easy understanding of the effect of global $\left(\Delta t, W_{p}\right)$ and local DSMC parameters $\left(\Delta x, T, \vec{u}, N_{\text {coll }}\right)$ on sample correlation.

\section{Acknowledgment}

The authors gratefully acknowledge financial support from the Department of Energy through grant DE-SC0001939.

\section{Appendix}

In this Appendix, we briefly discuss the flux approximation made in section 4 within the context of one dimensional cell in equilibrium. Two test cases (A and B) are examined which are summarized in Table 4. They correspond roughly to the conditions of cell 2 and 4 (shown in Fig. 2) of test case C64 (without considering $y$ velocities). At the start of each time step, the cell is populated by randomly placing $N^{k}$ (sampled from a Poisson distribution) particles in $[0, \Delta x]$ while sampling their velocity from a Maxwellian distribution. No collisions are performed and particles are moved during a time step of $\Delta t$. The number of particles leaving the cell at the interface located at $x=\Delta x$ during each step $\Delta t$ is recorded in $N_{\text {out }}^{k}$ which is the "actual" value plotted in Fig. 21. The predicted value of $N_{\text {out }}^{k}$ is that calculated from the value of $N^{k}$ at each step by Eq. (55) (reproduced below):

$$
\Delta N_{(i) \rightarrow(i+1)}^{k}=C_{(i) \rightarrow(i+1)} N_{i}^{k}
$$

where constant $C_{(i) \rightarrow(i+1)}$ is obtained via Eq. (62). By construction, and as demonstrated in Fig. (21), the correct expectation is obtained for the flux while its distribution in only approximately obtained. When $\frac{u_{x} \Delta t}{\Delta x}$ increases, the quality of the approximation of the distribution function greatly increases. Another consequence of using Eq. (117) is that it implicitly assumes that $\rho\left(N^{k}, N_{\text {out }}^{k}\right)=1$ which is not true as for instance $\rho\left(N^{k}, N_{\text {out }}^{k}\right) \approx 0.35$ and 0.1 for case A and B. When $\frac{u_{x} \Delta t}{\Delta x}$ increases, the flux of particles becomes more tied to the number of particles in the cell so that the correlation increases. The inaccuracy of the approximate method exposed in Section 4 is in large part due to this approximation. It is however the most natural approximation in order to obtain a simple analytical result and, in our view, acceptable given the results that are later obtained. Better results are surely obtainable by decomposing each cell in a series of subcells in the $x$ direction and using Eq. (117) for each subcell while suitably modifying the bounds of the integral of Eq. (57) for each. 


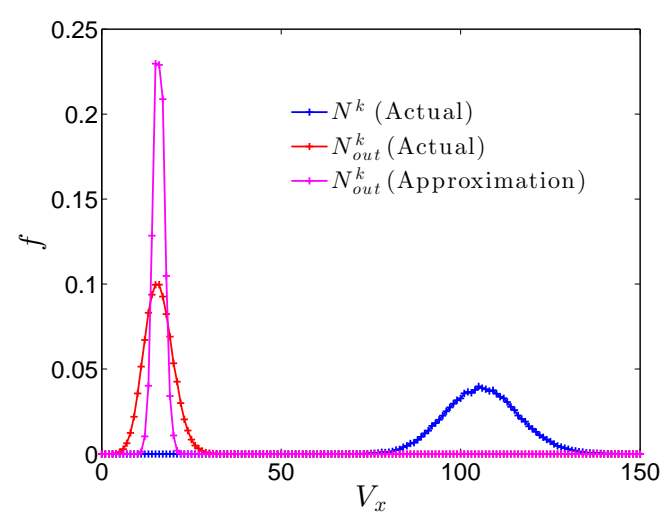

(a) Cell 2

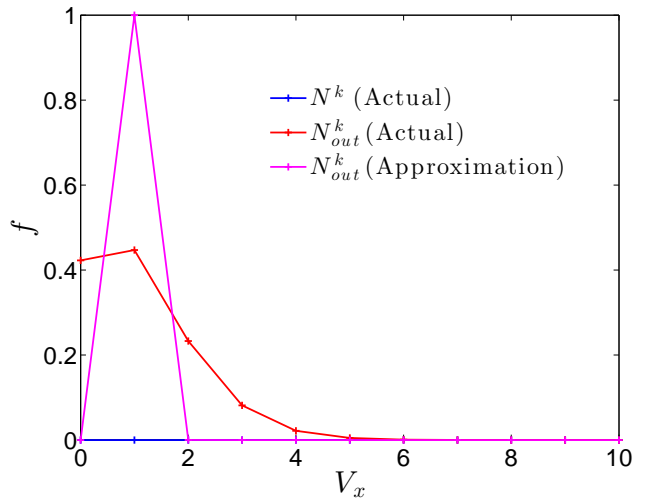

(b) Cell $4\left(N^{k}\right.$ distribution not shown)

Figure 21: Distribution function of the instantaneous number of particles in the cell $N^{k}$ and the number of particles moving out of the cell during $\Delta t: N^{k, \text { out }}$ observed and predicted by Eq. (62). 


\section{References}

[1] W. Wagner, A convergence proof for Bird's direct simulation Monte Carlo method for the Boltzmann equation, Journal of Statistical Physics 66 (1992) 1011-1044.

[2] N. G. Hadjiconstantinou, A. L. Garcia, M. Z. Bazant, G. He, Statistical error in particle simulations of hydrodynamic phenomena, Journal of Computational Physics 187 (2003) 274-297.

[3] M. Fallavollita, D. Baganoff, J. McDonald, Reduction of simulation cost and error for particle simulations of rarefied flows, Journal of Computational Physics 109 (1993) 30-36.

[4] G. Chen, I. D. Boyd, Statistical error analysis for the direct simulation Monte Carlo technique, Journal of Computational Physics 126 (1996) 434-448.

[5] I. D. Boyd, Monte Carlo simulation of nonequilibrium flow in a low-power hydrogen arcjet, Physics of Fluids 9 (1997) 3086.

[6] M. Wang, Z. Li, Gas mixing in microchannels using the direct simulation Monte Carlo method, International Journal of Heat and Mass Transfer 49 (2006) 1696-1702.

[7] T. Ferguson, A Course in Large Sample Theory, Chapman and Hall, 2004.

[8] J. Wu, K. Tseng, Analysis of micro-scale gas flows with pressure boundaries using direct simulation Monte Carlo method, Computers \& Fluids 30 (2001).

[9] M. N. Macrossan, C. R. Lilley, Viscosity of argon at temperatures $>2000$ K from measured shock thickness, Physics of Fluids 15 (2003) 3452 .

[10] Z. Sun, Z. Tang, Y. He, W. Tao, Proper cell dimension and number of particles per cell for DSMC, Computers \& Fluids $50(2011) 1-9$.

[11] M. Gallis, J. Torczynski, D. Rader, G. Bird, Convergence behavior of a new DSMC algorithm, Journal of Computational Physics 228 (2009) 4532-4548.

[12] E. P. Muntz, Rarefied gas dynamics, Annual Review of Fluid Mechanics 21 (1989) 387-422.

[13] J. M. Burt, I. D. Boyd, A hybrid particle approach for continuum and rarefied flow simulation, Journal of Computational Physics 228 (2009) $460-475$.

[14] T. E. Schwartzentruber, L. Scalabrin, I. D. Boyd, A modular particle-continuum numerical method for hypersonic nonequilibrium gas flows, Journal of Computational Physics 225 (2007) 1159 - 1174.

[15] G. Bird, Molecular Gas Dynamics and the Direct Simulation of Gas Flows, Oxford University Press, 1994.

[16] S. Dietrich, I. D. Boyd, Scalar and parallel optimized implementation of the direct simulation Monte Carlo method, Journal of Computational Physics 126 (1996) 328-342.

[17] A. L. Garcia, Direct simulation Monte Carlo: theory, methods, and open challenges, Proceedings of the Models and Computational Methods for Rarefied Flows NATO Conf. Proc. RTO-EN-AVT-194 , 2011.

[18] C. Galitzine, On the Accuracy and Efficiency of the Direct Simulation Monte Carlo Method, Ph.D. thesis, University of Michigan, 2014.

[19] A. Steland, Financial Statistics and Mathematical Finance: Methods, Models and Applications, Wiley, 2012.

[20] H. Flyvbjerg, H. G. Petersen, Error estimates on averages of correlated data, Journal of Chemical Physics 91 (1989) 461.

[21] J. Shanthikumar, U. Sumita, A central limit theorem for random sums of random variables, Operations Research Letters 3 (1984) $153-155$

[22] A. Cedilnik, K. Kosmelj, A. Blejec, The distribution of the ratio of jointly normal variables, Metodoloski zvezki 1 (2004) 99-108.

[23] G. Casella, R. Berger, Statistical Inference, Duxbury, 2001.

[24] T. Gombosi, Gaskinetic Theory, Cambridge University Press, 1994.

[25] C. Galitzine, I. D. Boyd, An adaptive procedure for the numerical parameters of a particle simulation, Journal of Computational Physics 281 (2015) $449-472$. 